\title{
Testing the white dwarf mass-radius relationship with eclipsing binaries
}

\author{
S. G. Parsons ${ }^{1 \star}$, B. T. Gänsicke 2 , T. R. Marsh $^{2}$, R. P. Ashley², M. C. P. Bours ${ }^{3}$ \\ E. Breedt ${ }^{2}$, M. R. Burleigh ${ }^{4}$, C. M. Copperwheat ${ }^{5}$, V. S. Dhillon ${ }^{1,6}$, M. Green ${ }^{2}$, \\ L. K. Hardy ${ }^{1}$, J. J. Hermes ${ }^{7}$, P. Irawati ${ }^{8}$, P. Kerry ${ }^{1}$, S. P. Littlefair ${ }^{1}$, \\ M. J. McAllister ${ }^{1}$, S. Rattanasoon ${ }^{1,7}$, A. Rebassa-Mansergas ${ }^{9}$, D. I. Sahman ${ }^{1}$ \\ and M. R. Schreiber 3,10 \\ 1 Department of Physics and Astronomy, University of Sheffield, Sheffield, S3 $7 R H, U K$ \\ 2 Department of Physics, University of Warwick, Coventry CV4 7AL, UK \\ 3 Instituto de Física y Astronomía, Universidad de Valparaíso, Avenida Gran Bretana 1111, Valparaíso, 2360102, Chile \\ 4 Department of Physics and Astronomy, University of Leicester, Leicester LE1 7RH, UK \\ 5 Astrophysics Research Institute, Liverpool John Moores University, IC2, Liverpool Science Park, L3 5RF, UK \\ 6 Instituto de Astrofísica de Canarias, Vía Lactea s/n, La Laguna, E-38205 Tenerife, Spain \\ 7 Hubble Fellow, Department of Physics and Astronomy, University of North Carolina, Chapel Hill, NC 27599-3255, USA \\ 8 National Astronomical Research Institute of Thailand, 191 Siriphanich Bldg., Huay Kaew Road, Chiang Mai 50200, Thailand \\ 9 Departament de Física, Universitat Politécnica de Catalunya, c/Esteve Terrades 5, 08860 Castelldefels, Spain \\ 10 Millenium Nucleus "Protoplanetary Disks in ALMA Early Science", Universidad de Valparaiso, Valparaiso 2360102, Chile
}

Accepted 2017 June 15. Received 2017 June 15; in original form 2017 February 27

\begin{abstract}
We present high precision, model independent, mass and radius measurements for 16 white dwarfs in detached eclipsing binaries and combine these with previously published data to test the theoretical white dwarf mass-radius relationship. We reach a mean precision of 2.4 per cent in mass and 2.7 per cent in radius, with our best measurements reaching a precision of 0.3 per cent in mass and 0.5 per cent in radius. We find excellent agreement between the measured and predicted radii across a wide range of masses and temperatures. We also find the radii of all white dwarfs with masses less than $0.48 \mathrm{M}_{\odot}$ to be fully consistent with helium core models, but they are on average 9 per cent larger than those of carbon-oxygen core models. In contrast, white dwarfs with masses larger than $0.52 \mathrm{M}_{\odot}$ all have radii consistent with carbonoxygen core models. Moreover, we find that all but one of the white dwarfs in our sample have radii consistent with possessing thick surface hydrogen envelopes $\left(10^{-5} \geq\right.$ $M_{\mathrm{H}} / M_{\mathrm{WD}} \geq 10^{-4}$ ), implying that the surface hydrogen layers of these white dwarfs are not obviously affected by common envelope evolution.
\end{abstract}

Key words: white dwarfs - binaries: eclipsing - stars: fundamental parameters stars: interiors

\section{INTRODUCTION}

The overwhelming majority of all stars born in the Galaxy will one day evolve into white dwarfs. White dwarfs are supported against collapse by electron degeneracy pressure and as such show the remarkable property that the more massive they are, the smaller their radius. Moreover, this massradius relationship sets an upper limit to the mass of a white

\footnotetext{
^ s.g.parsons@sheffield.ac.uk
}

dwarf (Chandrasekhar 1931) above which electron degeneracy can no longer support them, a result that underpins our understanding of type Ia supernovae and hence the expansion of the Universe (Riess et al. 1998; Perlmutter et al. 1999). The mass-radius relationship forms an essential part of many studies of white dwarfs, such as the initial-final mass relationship (e.g. Catalán et al. 2008), the white dwarf luminosity function (e.g. García-Berro \& Oswalt 2016) as well as allowing us to compute masses from spectroscopic data alone (e.g. Bergeron et al. 1992, 2001), and underlies the

(C) 2017 The Authors 
best mass measurements for white dwarfs in interacting binary systems (e.g. Wood et al. 1985; Littlefair et al. 2008; Savoury et al. 2011).

Despite its huge importance to a wide range of astrophysical topics, the white dwarf mass-radius relationship remains poorly tested observationally. Previous efforts involve using astrometric binaries where a white dwarf is paired with a bright main-sequence star and which have accurate distance measurements and dynamical masses from orbital fits. These can be combined with spectroscopic measurements of the gravitational redshift of the white dwarf to yield masses and radii (Shipman et al. 1997; Barstow et al. 2005; Bond et al. 2015). Unfortunately, very few of these systems have accurate orbital fits and so masses and radii are instead determined from a combination of parallax and spectroscopic data (e.g. Holberg et al. 2012). However, these measurements still rely on mass-radius relationships to a small extent, since they require an estimate of the intrinsic flux of the white dwarf (i.e. the monochromatic Eddington flux), made by fitting the spectrum of the white dwarf with model atmosphere codes (that in turn rely on a mass-radius relationship). This semi-empirical method can also be applied to isolated white dwarfs, provided they have accurate parallax measurements. Therefore, the large sample of parallax measurements from the Gaia mission should allow the detection of any offsets between observed and theoretical massradius relations. However, due to uncertainties in model atmospheres and evolutionary models, these results may be difficult to interpret and hence genuine model-independent mass and radius measurements are still required to properly test mass-radius relations and fully understand Gaia data (Tremblay et al. 2017).

Using isolated white dwarfs, or those in wide astrometric binaries, limits the range over which one can test the white dwarf mass-radius relationship. This is because all of these white dwarfs will have masses larger than $\sim 0.5 \mathrm{M}_{\odot}$, since lower mass white dwarfs are produced from mainsequence stars with masses less than $\sim 0.8 \mathrm{M}_{\odot}$ and the Universe is not old enough for these stars to have evolved off the main-sequence yet. However, white dwarfs with masses less than $0.5 \mathrm{M}_{\odot}$ have been identified, and are almost exclusively found in close binary systems, the low masses being the result of past interactions between the two stars (Marsh 1995; Rebassa-Mansergas et al. 2011), although note that massive white dwarfs can also be found in close binaries (see the latest catalogue from Rebassa-Mansergas et al. 2016, for example). Moreover, a fraction of these close binaries are eclipsing, allowing us to directly measure the mass and radius of the white dwarf to a precision of $1-2$ per cent, independent of model atmosphere calculations. Double-lined eclipsing binaries are generally the best sources for mass-radius measurements (Torres et al. 2010).

White dwarfs with masses below $0.5 \mathrm{M}_{\odot}$ are expected to have cores composed primarily of helium, since they are the result of strong mass-loss episodes during the red giant branch stage (e.g. from binary interactions), before the helium core flash has converted the core to carbon and oxygen. However, it may still be possible to form carbon-oxygen $(\mathrm{C} / \mathrm{O})$ core white dwarfs with masses as low as $0.33 \mathrm{M}_{\odot}$ via anomalous mass-loss episodes on the red giant branch or the core He-burning phase (Prada Moroni \& Straniero 2009), meaning that both $\mathrm{C} / \mathrm{O}$ and $\mathrm{He}$ core white dwarfs may exist in this mass range. Since He core white dwarfs are more expanded than those with $\mathrm{C} / \mathrm{O}$ cores of the same mass and temperature (e.g. Panei et al. 2007), precise enough radius measurements can distinguish between the two possible core compositions.

In this paper we present precise mass and radius measurements for 16 white dwarfs in detached eclipsing binaries with low-mass main-sequence star companions and combine them with 10 previous measurements to test theoretical mass-radius relationships over a wide range of masses and temperatures. Results for the main-sequence stars will be presented elsewhere.

\section{OBSERVATIONS AND THEIR REDUCTION}

In this section we describe our photometric and spectroscopic observations. Due to the large number of entries, the observing logs can be found in Table A1 (photometry) and Table B1 (spectroscopy) in the appendix.

\section{$2.1 \quad$ ULTRACAM photometry}

We obtained high-speed photometry for the majority of our targets using the frame-transfer CCD camera ULTRACAM (Dhillon et al. 2007). Observations were performed between 2002 and 2016 and were obtained with ULTRACAM mounted as a visitor instrument on the 3.5-m New Technology Telescope (NTT) on La Silla, Chile, the 4.2-m William Herschel Telescope (WHT) on La Palma, Spain and the 8.2-m Very Large Telescope (VLT) at Paranal, Chile. ULTRACAM uses a triple beam setup allowing one to obtain data in the $u^{\prime}, g^{\prime}$ and either $r^{\prime}$ or $i^{\prime}$ band simultaneously.

All of these data were reduced using the ULTRACAM pipeline software. The source flux was determined with aperture photometry using a variable aperture scaled according to the full width at half maximum (FWHM). Variations in observing conditions were accounted for by determining the flux relative to a comparison star in the field of view. The data were flux calibrated using observations of standard stars observed during twilight.

\subsection{ULTRASPEC photometry}

We observed a number of eclipsing systems with the highspeed camera ULTRASPEC (Dhillon et al. 2014) mounted on the 2.4-m Thai National Telescope (TNT) on Doi Inthanon, Thailand. ULTRASPEC is a high-speed frametransfer EMCCD camera that operates in a similar fashion to ULTRACAM but with a single beam. Our observations were made using either the $g^{\prime}$ band filter or a broad $u^{\prime}+g^{\prime}+r^{\prime}$ filter known as KG5 (as described in Dhillon et al. 2014, see also the appendix of Hardy et al. 2017). The ULTRASPEC data were reduced using the ULTRACAM pipeline as previously described.

\subsection{X-shooter spectroscopy}

We spectroscopically observed our eclipsing systems with the medium resolution echelle spectrograph X-shooter (Vernet et al. 2011), which is mounted at the Cassegrain focus of the VLT-UT2 at Paranal, Chile. X-shooter covers the 

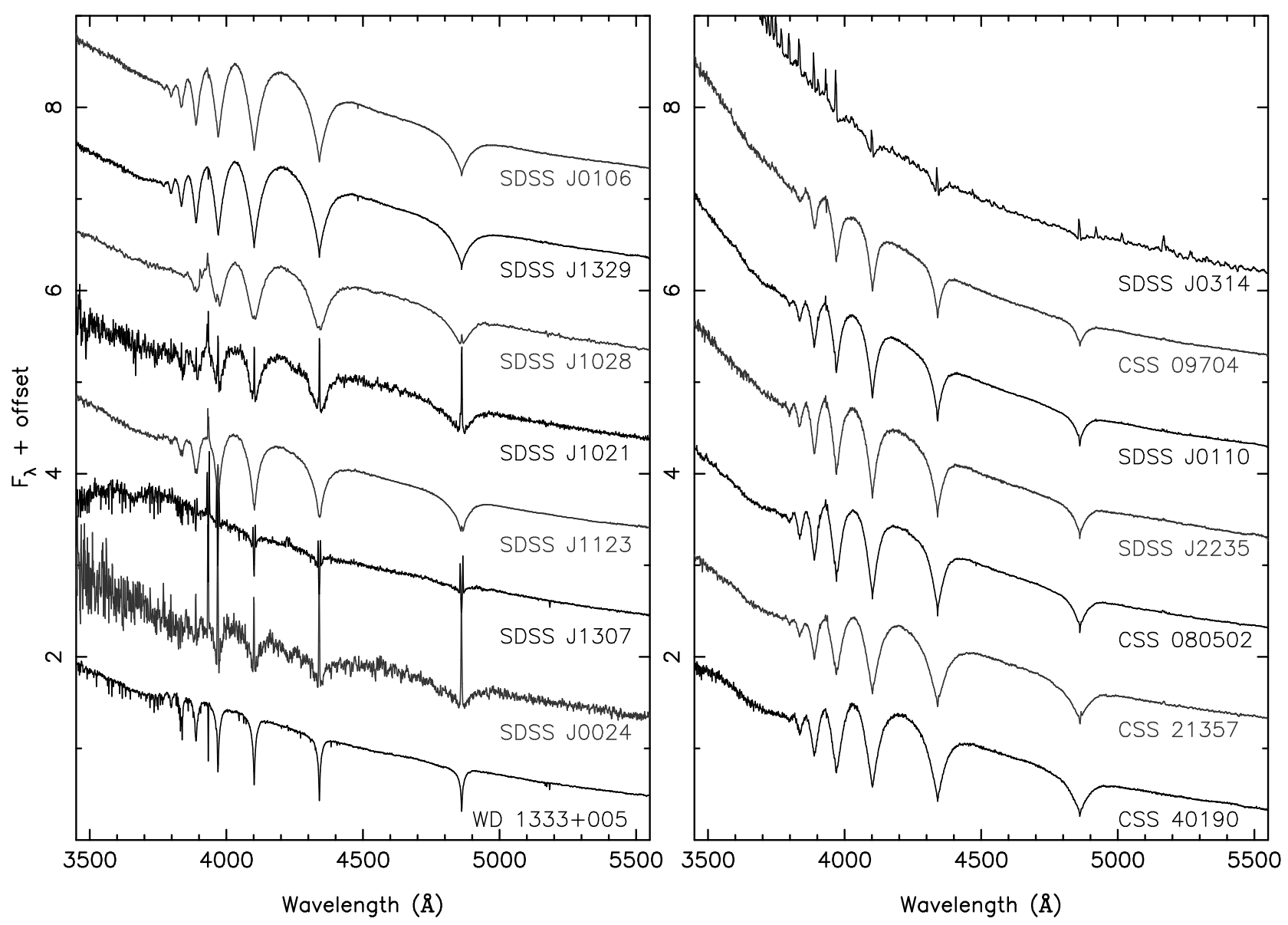

Figure 1. X-shooter UVB arm spectra of the white dwarfs in our binaries ordered from coolest (bottom-left) to hottest (top-right), in the white dwarf restframes. The $\mathrm{M}$ dwarf components have been subtracted from these spectra, though any strong, variable emission lines from them (e.g. from irradiation or activity) are not completely removed (particularly strong in the case of SDSS J0314+0206). The spectra have been binned by a factor of 10 for clarity.

spectral range from the atmospheric cutoff in the UV to the near-infrared $\mathrm{K}$ band in three separate arms, known as the UVB (0.30-0.56 microns), VIS (0.56-1.01 microns) and NIR (1.01-2.40 microns). Separate slit widths can be set for each arm and the UVB and VIS arms can be binned by up to a factor of $2 \times 2$. All our observations were performed with slit widths of 1.0", 0.9 " and 0.9 " in the UVB, VIS and NIR arms respectively. During X-shooter exposures the evolution of the parallactic angle is not followed. Prior to 2012 the atmospheric dispersion corrector (ADC) allowed observations to be taken in long series without needing to reorient the slit. The ADCs were disabled in August 2012 and hence subsequent observations had the potential to suffer from large slit losses, particularly for a long series of exposures. This effect was mitigated by setting the slit angle to pass through the parallactic halfway through a 1 hour set of exposures, re-aligning the slit after each hour, to ensure that it never drifted too far from the parallactic. In addition, for all observations the VIS arm was binned by a factor of 2 in the spatial direction, while the UVB arm was also binned by a factor of 2 in the spatial direction (for all observations) and by a factor of 2 in the dispersion direction just for our faintest targets $(g>19)$. This results in a resolution of $R \sim 7500$.

In addition to our main targets, we also observed a num- ber of $\mathrm{M}$ dwarf template stars with the same instrumental setup (from M1.0 to M8.0 in steps of 0.5 of a spectral class) as well as several bright DC white dwarfs, which we used to remove telluric features from our target spectra. All of the data were reduced using the standard pipeline release of the $\mathrm{X}$-shooter Common Pipeline Library (CPL) recipes (version 2.6.8) within ESORex, the ESO Recipe Execution Tool.

For a small number of our targets the NIR arm data had very low signal-to-noise ratios due to the faintness of these targets. These are indicated in Table B1 and we discarded these data from our subsequent analysis. Figure 1 shows UVB arm spectra for all of our targets with the M dwarf components removed (using in-eclipse or appropriately scaled template spectra, see Section 5.1).

The accuracy of the wavelength calibration of X-shooter data from the pipeline reduction is $0.03 \mathrm{~nm}$ in the UVB, $0.02 \mathrm{~nm}$ in the VIS and $0.004 \mathrm{~nm}$ in the NIR arm, corresponding to a velocity precision of $\sim 1 \mathrm{~km} \mathrm{~s}^{-1}$ at $\mathrm{H} \alpha$. Additionally, there is a further $\sim 0.01 \mathrm{~nm}$ offset in the wavelength solution due to imperfect positioning of the target in the slit ${ }^{1}$. How-

1 https://www.eso.org/sci/facilities/paranal/instruments/xshoo ter/doc/XS_wlc_shift_150615.pdf 
ever, the wavelength accuracy can be improved in the VIS and NIR arms using sky emission and telluric lines, enabling velocity measurements to an accuracy of $\sim 0.5 \mathrm{~km} \mathrm{~s}^{-1}$. Since there are no sky lines in the UVB arm we used any mainsequence star lines visible in the UVB arm to measure any systemic velocity offsets (relative to the main-sequence star features in the VIS/NIR arms). In some cases there are no such features visible, in which case we consider the accuracy of any velocity measurements in the UVB arm to be $1 \mathrm{~km} \mathrm{~s}^{-1}$. We find no evidence of variations in the wavelength shifts between subsequent spectra of the same target, therefore, this primarily affects the precision of our systemic velocity measurements, rather than the radial velocity amplitudes.

\section{BREAKING THE DEGENERACY BETWEEN INCLINATION AND SCALED RADII}

Observations of just the eclipse of the white dwarf by its main-sequence star companion do not contain enough information to fully solve for the binary parameters. This is because the eclipse profile only contains two pieces of information (its width and the duration of the ingress/egress), while there are three unknowns: the orbital inclination, $i$, and the radii of the two stars scaled by the orbital separation $\left(R_{\mathrm{WD}} / a\right.$ and $\left.R_{\mathrm{MS}} / a\right)$. Therefore, it is possible to fit the same eclipse profile with a high inclination system containing a large white dwarf and small main-sequence star or a lower inclination system with a smaller white dwarf and larger main-sequence star. Hence at least one more piece of information is required in order to break this degeneracy. In this section we outline several different techniques that we use to this end.

\subsection{The depth of the secondary eclipse}

The most straightforward and direct method of breaking the degeneracy between the inclination and scaled radii is to measure the depth of the secondary eclipse (i.e. the transit of the white dwarf across the face of its main-sequence star companion). This is analogous to an exoplanet transit and hence the depth of the eclipse is the ratio of the areas of the two stars, $\left(R_{\mathrm{WD}} / R_{\mathrm{MS}}\right)^{2}$. Therefore, combining this with the primary eclipse profile directly yields the inclination and two radii (see Parsons et al. 2010, for an example of this technique).

However, since white dwarfs are so much smaller than main-sequence stars and because the white dwarf dilutes the eclipse, the depth of the secondary eclipse is usually less than 1-2 per cent. Given the relative faintness of these objects (the mean $g$ band magnitude of our sample is 17.6), in the vast majority of systems, detecting and measuring the depth of the secondary eclipse is currently not possible and other techniques are required. The secondary eclipse is only detected in one of our systems, RR Cae.

\subsection{The gravitational redshift of the white dwarf}

General relativity tells us that the light emerging from the gravitational well of a white dwarf is redshifted by an

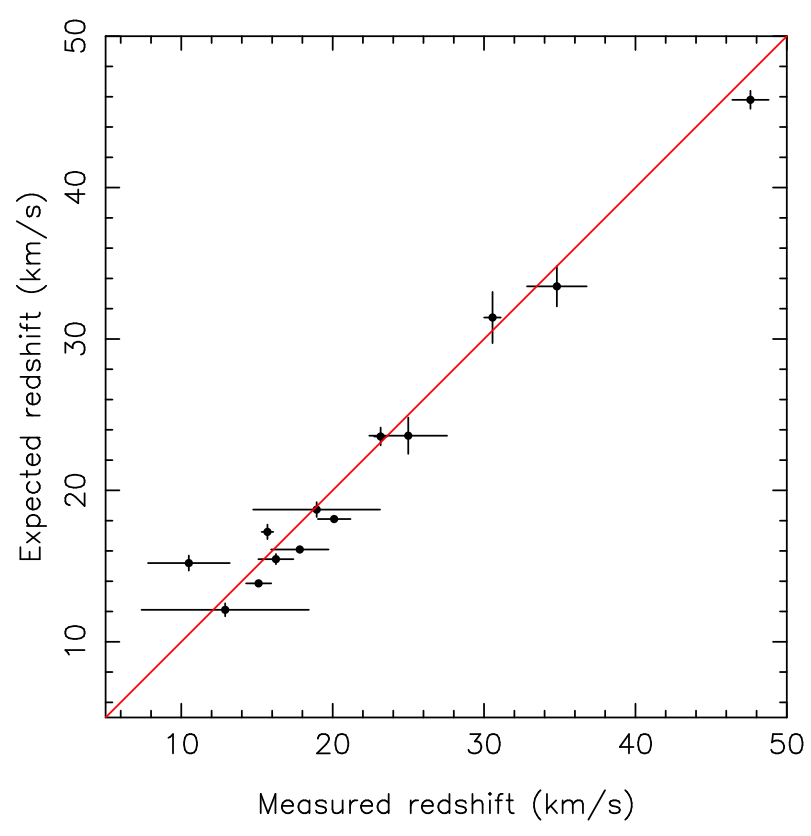

Figure 2. The measured and computed gravitational redshifts of the white dwarfs in our binaries. The expected redshift values are those determined from the mass and radius of the white dwarf, computed from Equation 1. The measured values are the spectroscopic redshifts from our X-shooter data. Only objects where the spectroscopic redshift was not used to determine the inclination are shown.

amount given by

$V_{z}=0.635\left(M_{\mathrm{WD}} / M_{\odot}\right)\left(R_{\odot} / R_{\mathrm{WD}}\right) \mathrm{km} \mathrm{s}^{-1}$.

Furthermore, if we know the radial velocity amplitudes of the two stars then Kepler's third law tells us

$M_{\mathrm{WD}}=\frac{P K_{\mathrm{MS}}\left(K_{\mathrm{WD}}+K_{\mathrm{MS}}\right)^{2}}{2 \pi G \sin ^{3} i}$,

where $P$ is the orbital period and $K_{\mathrm{WD}}$ and $K_{\mathrm{MS}}$ are the radial velocity semi-amplitudes of the white dwarf and mainsequence star respectively. Therefore, if we can spectroscopically measure the gravitational redshift of the white dwarf (measured from the offset in the radial velocity semiamplitudes of the two stars), then combining these two equations gives us a relationship between the white dwarf's radius and the binary inclination. This can then be used in combination with the primary eclipse profile to break the degeneracy between the inclination and scaled radii of the two stars. See Parsons et al. (2012b) for an example of this technique.

The advantage of this technique is that all it requires is that spectral features from both stars are visible. Therefore, it is applicable to a wide range of systems. However, the precision of the final parameters is strongly correlated to how well the radial velocity semi-amplitudes of the two stars can be measured and therefore it can be less precise than some of the other methods, particularly in systems where one star strongly dominates over the other in the spectrum.

Finally, we note that there has been some discrepancy between the measured and expected gravitational redshift of the white dwarf in the wide binary Sirius (Barstow et al. 2005) and several previous redshift measurements of white 
dwarfs in close binaries were only marginally in agreement with the expected value (Maxted et al. 2007; Parsons et al. 2010; Pyrzas et al. 2012). Additionally, in several cases, we use features from the white dwarf that originate from accreting material from the wind of the main-sequence star (and may form higher up in the white dwarf's atmosphere, hence lower gravitational potential, particularly so in the case of emission lines from the white dwarf). Therefore, it is worth checking the accuracy of this method. In Figure 2 we show the measured and expected redshift values for all of the white dwarfs in our sample (and previously published) in which the gravitational redshift itself was not used to determine the stellar parameters (i.e. the method outlined above was not used). The values are in excellent agreement, even when using lines originating from accreting wind material. Therefore, stellar parameters determined from this technique should be accurate and also shows that our final mass and radius constraints are consistent regardless of the method used to break the inclination degeneracy.

\subsection{The rotational broadening of the $M$ star}

The short periods and extreme mass ratios of these binaries means that the main-sequence star components are tidally locked to the white dwarf and therefore their rotational period matches the orbital period (for low-mass M stars in binaries with a white dwarf and periods of less than a day, the tidal synchronisation timescale is of the order of $10^{5}$ years and so all of our systems should be synchronised Zahn 1977). This means that these stars are rotating quite rapidly and hence their spectral lines are broadened by a factor given by

$V_{\mathrm{rot}} \sin i=K_{\mathrm{MS}}(1+q) \frac{R_{\mathrm{MS}}}{a}$,

where $q=M_{\mathrm{WD}} / M_{\mathrm{MS}}$, the binary mass ratio (Marsh et al. 1994). The rotational broadening can be measured from spectroscopy by artificially broadening the lines of template stars to fit the observed line profiles of our systems, taking into account any additional smearing of the lines from the velocity shift of the main-sequence star during an exposure (see Marsh et al. 1994, for details of this method). Note that we applied a high-pass filter to both the observed and broadened template spectra before comparing them in order to prevent the continuum dominating the rotational broadening determination. If both $K_{\mathrm{MS}}$ and $q$ have also been spectroscopically measured, then the rotational broadening gives a direct measurement of the scaled radius of the main-sequence star, which can be combined with the primary eclipse profile to fully determine the binary and stellar parameters (see Parsons et al. 2016, for an example of this technique).

This technique is useful for systems in which the mainsequence star contributes a substantial fraction of the optical flux. Moreover, it is best suited to systems with very short orbital periods and those that are close to Roche-lobe filling, where the rotational broadening is maximised. However, in systems where the white dwarf completely dominates the optical flux, or where the main-sequence star is strongly irradiated by the white dwarf, this technique may not be reliable or even possible.

\subsection{The amplitude of ellipsoidal modulation}

The tidal distortion of main-sequence stars in close binaries with white dwarfs causes a sinusoidal variation in the light curve on half the binary period, caused by the variation in surface area that the star presents to the Earth during its orbit. The amplitude of this effect is approximately given by

$\frac{\delta F}{F}=0.15 \frac{\left(15+u_{\mathrm{MS}}\right)\left(1+\beta_{\mathrm{MS}}\right)}{3-u_{\mathrm{MS}}}\left(\frac{R_{\mathrm{MS}}}{a}\right)^{3} q \sin ^{2} i$,

where $u_{\mathrm{MS}}$ is the linear limb darkening coefficient and $\beta_{\mathrm{MS}}$ is the gravity darkening exponent of the main-sequence star (Morris \& Naftilan 1993; Zucker et al. 2007); note that the companion must be tidally locked for this assumption to be valid. Therefore, this effect can be used to establish a relationship between the scaled radius of the main-sequence star and the inclination in a manner independent of the primary eclipse profile, and hence can be combined to break the degeneracy between these parameters (see Parsons et al. 2012c, for an example of this technique).

However, while this technique can work in some systems, it is often difficult to reliably measure the ellipsoidal amplitude due to the unknown contribution from starspots, which may dilute or strengthen the ellipsoidal amplitude depending upon their location on the surface of the star. Therefore, in this paper we do not use this technique to help break the degeneracy between the scaled radii and inclination. However, when visible in the light curve, we do check that the amplitude is consistent with our best-fit models.

\section{RADIAL VELOCITY MEASUREMENTS}

We measured the radial velocity semi-amplitudes of both stars in each binary by first identifying the cleanest features from each star then fitting them with a combination of a straight line and a Gaussian (or double Gaussian in the case of Balmer absorption from the white dwarf, that includes a broader component for the wings of the line and a narrower one for the core of the line). Each spectrum was fitted individually and the resulting velocity measurements $\left(v_{t}\right)$ were combined to determine the semi-amplitude $(K)$ and systemic velocity $(\gamma)$ of each star by fitting them with the following equation:

$v_{t}=\gamma+K \sin \left(\frac{2 \pi\left(t-t_{0}\right)}{P}\right)$,

where $P$ is the orbital period and $t_{0}$ is the mid-eclipse time (previously determined from the photometry). The results for all systems are listed in Table 1, which also gives the specific features used to measure the velocities of each star, as well as the rotational broadening (where possible) for the main-sequence stars measured as outlined in Section 3.3. Recall that the difference in the $\gamma$ values between the white dwarf and main-sequence star is related to the gravitational redshift of the white dwarf. We also show the radial velocity curves for all our X-shooter targets in Figure 3 (note that the system RR Cae is not included in this figure, since the radial velocity data have already been published in Ribeiro et al. 2013).

For some of our objects, specifically those containing very hot white dwarfs, the main-sequence star is irradiated to such an extent that its spectral features are completely 


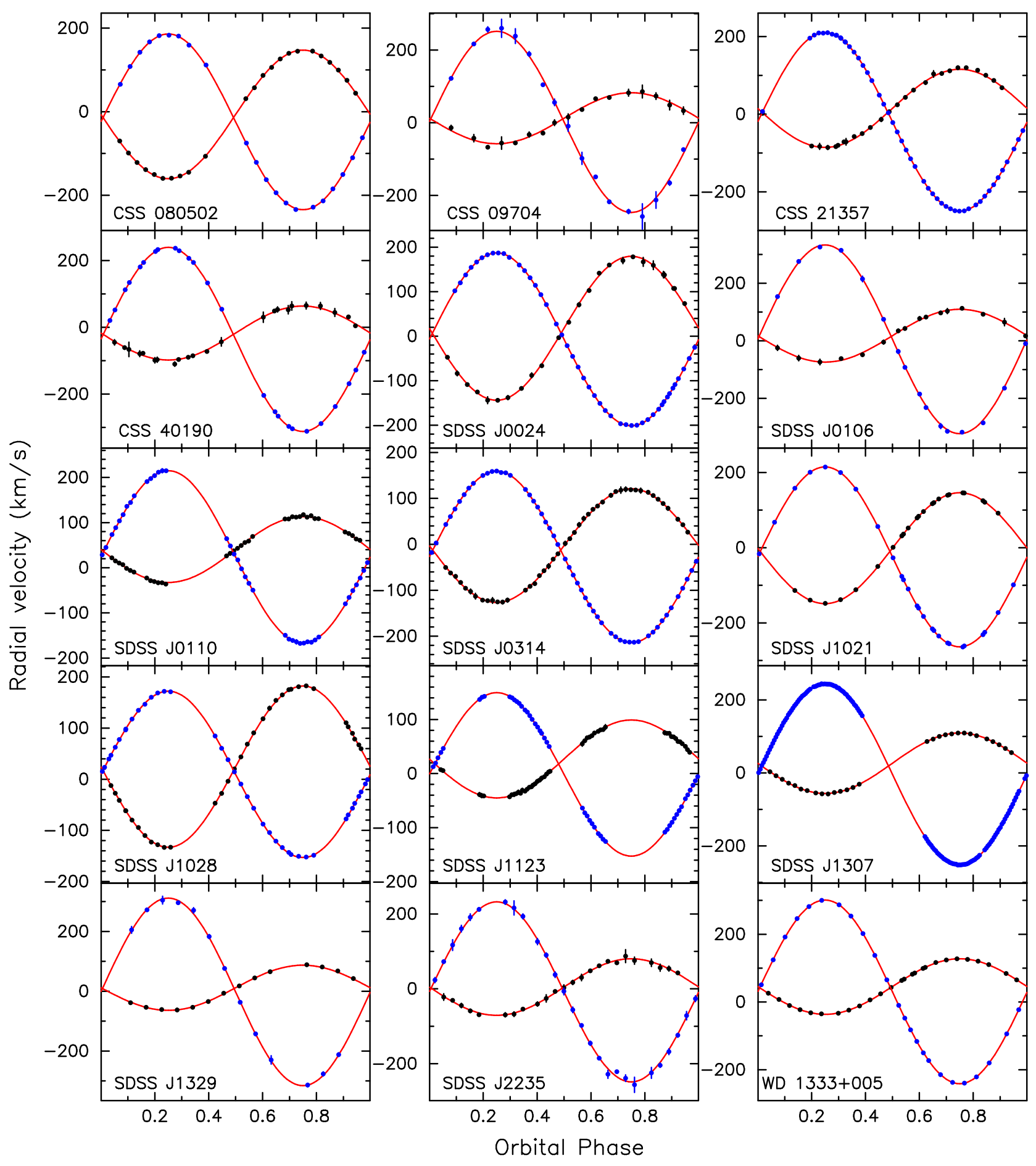

Figure 3. Radial velocity measurements for the white dwarfs (black) and main-sequence stars (blue) for all the binaries we observed with X-shooter (note that RR Cae was not observed with X-shooter). The best fit curves are shown in red.

diluted. In these cases, many absorption lines from the mainsequence star are instead in emission. However, while these emission lines can be strong and hence give very precise velocity measurements, because they arise from the heated face of the star they do not track the centre-of-mass of the star, but rather the centre-of-light of the emission region on the hemisphere facing the white dwarf. Therefore, they are not ideal features to use for precise stellar parameter measurements. However, in some cases these emission lines are the only features visible from the main-sequence star. It is possible to correct from the emission line velocity $\left(K_{\text {emis }}\right)$ to the centre-of-mass velocity via an equation,

$K_{\mathrm{MS}}=\frac{K_{\mathrm{emis}}}{1-f(1+q) \frac{R_{\mathrm{MS}}}{a}}$,

where $f$ is a constant between 0 and 1 which depends upon 
Table 1. Spectroscopic measurements. We have highlighted with an asterisk $\left(^{*}\right)$ those systems where the radial velocity of the mainsequence star was determined by applying a correction factor to the velocity of emission lines (see Section 4 ). Data for RR Cae were taken from Ribeiro et al. (2013). Note that when referring to the hydrogen absorption lines of white dwarfs we actually fitted the narrow core of the line, rather than the broader wings.

\begin{tabular}{|c|c|c|c|c|c|c|c|}
\hline Object & $\begin{array}{c}K_{\mathrm{WD}} \\
\left(\mathrm{km} \mathrm{s}^{-1}\right)\end{array}$ & $\begin{array}{c}\gamma_{\mathrm{WD}} \\
\left(\mathrm{km} \mathrm{s}^{-1}\right)\end{array}$ & $\begin{array}{l}\text { WD feature } \\
\text { used }\end{array}$ & $\begin{array}{c}K_{\mathrm{MS}} \\
\left(\mathrm{km} \mathrm{s}^{-1}\right)\end{array}$ & $\begin{array}{c}\gamma_{\mathrm{MS}} \\
\left(\mathrm{km} \mathrm{s}^{-1}\right)\end{array}$ & $\begin{array}{l}\text { MS feature } \\
\text { used }\end{array}$ & $\begin{array}{l}V_{\text {rot }} \sin i \\
\left(\mathrm{~km} \mathrm{~s}^{-1}\right)\end{array}$ \\
\hline CSS 080502 & $153.89 \pm 0.99$ & $-6.51 \pm 0.65$ & $\mathrm{H} \beta$ & $210.33 \pm 0.43$ & $-24.34 \pm 1.79$ & Na I 8183/94 & $131.76 \pm 2.56$ \\
\hline CSS 09704 & $70.2 \pm 5.1$ & $12.5 \pm 4.9$ & $\mathrm{H} \alpha$ & $249.1 \pm 7.4^{*}$ & $2.5 \pm 6.8$ & $\mathrm{H} \alpha(\mathrm{em})$ & - \\
\hline CSS 21357 & $100.63 \pm 2.51$ & $15.06 \pm 1.96$ & $\mathrm{H} \beta$ & $230.27 \pm 0.50$ & $-19.75 \pm 0.50$ & $\mathrm{Na}$ I $8183 / 94$ & $60.78 \pm 2.06$ \\
\hline CSS 40190 & $80.85 \pm 4.74$ & $-17.09 \pm 4.09$ & $\mathrm{H} \beta$ & $275.92 \pm 0.88$ & $-36.03 \pm 0.92$ & Na I $8183 / 94$ & $74.75 \pm 1.45$ \\
\hline RR Cae & $73.3 \pm 0.4$ & $98.0 \pm 1.0$ & $\mathrm{H} \beta$ & $195.1 \pm 0.3$ & $77.9 \pm 0.4$ & $\mathrm{H} \alpha$ & - \\
\hline SDSS J0024+1745 & $161.67 \pm 3.17$ & $17.82 \pm 2.45$ & Ca II 3934 & $194.30 \pm 0.20$ & $-7.17 \pm 0.77$ & Na I $8183 / 94$ & $107.42 \pm 1.80$ \\
\hline SDSS J0106-0014 & $91.86 \pm 3.49$ & $17.83 \pm 3.44$ & $\mathrm{H} \alpha$ & $328.15 \pm 3.22$ & $5.04 \pm 3.16$ & Na I $8183 / 94$ & - \\
\hline SDSS J0110+1326 & $72.90 \pm 1.29$ & $40.36 \pm 0.78$ & $\mathrm{H} \alpha$ & $190.85 \pm 1.23$ & $24.11 \pm 0.86$ & K I 12522 & $74.20 \pm 4.40$ \\
\hline SDSS J0314+0206 & $123.69 \pm 2.04$ & $-2.81 \pm 1.35$ & He I 5876 & $186.77 \pm 0.50$ & $-27.31 \pm 0.58$ & K I 12522 & - \\
\hline SDSS J1021+1744 & $147.45 \pm 0.60$ & $-1.20 \pm 0.50$ & $\mathrm{H} \alpha(\mathrm{em})$ & $239.21 \pm 0.70$ & $-24.37 \pm 0.50$ & Na I $8183 / 94$ & $140.56 \pm 2.26$ \\
\hline SDSS J1028+0931 & $157.75 \pm 1.00$ & $24.64 \pm 0.80$ & $\mathrm{H} \alpha(\mathrm{em})$ & $162.68 \pm 0.50$ & $9.53 \pm 0.50$ & Na I $8183 / 94$ & $90.38 \pm 1.50$ \\
\hline SDSS J1123-1155 & $72.09 \pm 1.39$ & $27.07 \pm 0.90$ & Ca II 3934 & $151.17 \pm 0.20$ & $-1.44 \pm 0.50$ & Na I $8183 / 94$ & - \\
\hline SDSS J1307+2156 & $82.80 \pm 0.59$ & $26.59 \pm 0.50$ & Ca II 3934 & $247.89 \pm 0.28$ & $-3.97 \pm 0.50$ & K I 12522 & $54.56 \pm 2.56$ \\
\hline SDSS J1329+1230 & $75.50 \pm 1.70$ & $11.72 \pm 1.87$ & Ca II 3934 & $313.9 \pm 5.8^{*}$ & $-2.03 \pm 1.20$ & $\mathrm{H} \alpha(\mathrm{em})$ & - \\
\hline SDSS J2235+1428 & $75.52 \pm 4.74$ & $4.75 \pm 3.43$ & $\mathrm{H} \alpha$ & $240.56 \pm 4.64$ & $-8.15 \pm 4.34$ & Na I $8183 / 94$ & $65.86 \pm 8.50$ \\
\hline WD 1333+005 & $81.95 \pm 0.47$ & $45.67 \pm 0.50$ & Ca II 3934 & $271.19 \pm 0.20$ & $29.98 \pm 0.50$ & NaI $8183 / 94$ & $70.56 \pm 2.06$ \\
\hline
\end{tabular}

the location of the centre of light (Parsons et al. 2012a). For $f=0$ the emission is spread uniformly across the entire surface of the secondary star and therefore the centre of light is the same as the centre of mass. For $f=1$ all of the flux is assumed to come from the point on the mainsequence star's surface closest to the white dwarf (the substellar point). This sets the upper and lower limits on the true centre-of-mass velocity. However, we can be more precise than this, since this $f$ factor is related to the optical depth of the emission (Parsons et al. 2010). With a sufficient number of emission lines from different atomic species it is possible to reliably estimate the centre-of-mass radial velocity to within $\sim 5 \mathrm{~km} \mathrm{~s}^{-1}$. This technique was tested by Parsons et al. (2012b) for SDSS J1212-0123, where both absorption and emission features are present.

Finally, there are some systems where the irradiation is fairly mild and so absorption lines from the main-sequence star are still visible, but there is a subtle emission core to the line that slightly shifts the velocity of the line. In these cases it is important to test if this is having a noticeable effect by measuring the equivalent width of the line as a function of orbital phase. If the line is weaker when the heated side of the star is viewed face on then the velocity will not be reliable. We did this for all the lines we used to measure the radial velocity of the main-sequence star and found that they were clean and reliable (i.e. showed no variations in their equivalent widths throughout the orbit).

\section{SPECTRAL FITTING}

In this section we detail how we removed the main-sequence star features from our spectra and how the resulting white dwarf spectra were then fitted to determine their effective temperatures.

\subsection{Main-sequence star removal}

In order to accurately fit the spectra of the white dwarfs in our binaries we need to remove the main-sequence star contributions. This is important since fitting spectra with both white dwarf and main-sequence star models simultaneously has been shown to give erroneous results when the main-sequence star is particularly dominant (Parsons et al. 2013) and reliable white dwarf effective temperature measurements are essential so that we can compare our mass and radius measurements with the correct models.

We spectroscopically observed all of our X-shooter targets during the eclipse of the white dwarf in order to obtain clean spectra of just the main-sequence star components. For the majority of our targets this was successful, however, for four of our objects (CSS 09704, SDSS J0106-0014, SDSS J1329+1230 and SDSS J2235+1428) the in-eclipse spectra had extremely low signal-to-noise ratios $(\mathrm{S} / \mathrm{N}<1)$ due to a combination of the faintness of the main-sequence components and the short duration of the eclipse limiting the exposure time. We then used these in-eclipse spectra (or template spectra in the case of the four low $\mathrm{S} / \mathrm{N}$ objects, determined from their measured masses and the mass-spectral class relationship from Baraffe \& Chabrier 1996) and subtracted them from all other spectra after shifting them into the main-sequence star's rest frame. We then shifted these main-sequence star subtracted spectra into the rest frame of the white dwarf and averaged them to create the spectra shown in Figure 1.

While this process removes the absorption features of the main-sequence stars, the majority of emission lines were not removed, since these tend to be highly variable as they arise from a combination of irradiation and stellar activity, hence these are still present in the final spectra. For example, the highly irradiated main-sequence star in SDSS J0314+0206 produces a large number of emission lines as seen in Figure 1. Furthermore, in systems where the mainsequence star is highly irradiated, or very close to filling its 
Roche lobe, the absorption features can vary in strength with orbital phase due to temperature variations over the surface of the star, hence the subtraction is never perfect and we therefore limit our spectral analysis to the shortest wavelength data (the UVB arm of X-shooter) where the white dwarfs generally dominate the flux anyway, to reduce this effect as much as possible.

\subsection{White dwarf spectral fitting}

The temperature of a white dwarf can have a significant effect on its radius, in particular for relatively hot, lowmass white dwarfs. We fitted the X-Shooter spectra of the white dwarfs in our sample following the method outlined in Rebassa-Mansergas et al. (2007). The X-shooter spectra of each white dwarf were shifted into its rest-frame, and had the contribution of the M-dwarf removed (see Section 5.1 and Figure 1). We used an updated model grid of hydrogen (DA) atmosphere white dwarfs computed with the code described in Koester (2010), adopting ML2/ $\alpha=0.8$ for the mixing length in convective atmospheres $\left(T_{\text {eff }}<15,000 \mathrm{~K}\right)$. The continuum-normalised Balmer lines in the X-Shooter spectra were fitted with the full model grid, and the bestfit parameters were then determined from a bicubic fit to the $\chi^{2}$ surface in the $\left(T_{\text {eff }}, \log g\right)$ plane. The degeneracy between the "hot" and "cold" solution, corresponding to similar equivalent widths of the Balmer lines, was resolved using the slope of the white dwarf continuum.

To test the robustness of the fits, and estimate realistic parameters, we fitted both the average and all individual $\mathrm{X}$-Shooter spectra. The average spectra were of high signalto-noise ratio $(\mathrm{S} / \mathrm{N} \simeq 60-140$, apart for SDSS J0024+1745 $(\mathrm{S} / \mathrm{N} \simeq 12)$ and $\mathrm{SDSS} J 1021+1744(\mathrm{~S} / \mathrm{N} \simeq 40))$, and uncertainties in the fits to these spectra are therefore dominated by systematic effects. In contrast, the fits to the individual X-Shooter spectra (typical $\mathrm{S} / \mathrm{N} \simeq 10-20$ ) are dominated by the statistical uncertainties. We compute the bestfit parameters for each system as the average from the fits to the individual spectra, and adopt as uncertainties the standard deviations from these mean values. For all systems, the fit parameters derived from the average spectrum are, within the uncertainties, consistent with these bestfit values (see Tremblay et al. 2017 for a detailed discussion on the precision of atmospheric parameters measured from multiple spectra). The X-Shooter spectra (average and individual) of SDSSJ1021+1744, SDSS J0024+1745, and SDSS J0314+0206 were significantly contaminated by emission lines from the active and/or irradiated companion star. We masked the regions affected by the emission lines before fitting the spectra, and the atmospheric parameters of these stars are subject to larger uncertainties.

In the case of SDSS J1307+2156, the Balmer lines were so weak and affected by emission lines that the fitting procedure failed. Instead we fitted the average X-Shooter spectrum (this time including the M-dwarf contribution), supplemented by the SDSS ugriz fluxes and the GALEX near-ultraviolet flux using a composite spectrum of a DA white dwarf model, with the surface gravity fixed $\log g=$ 8.062 , as determined from the light curve fits. We found that the spectral energy distribution of the white dwarf in SDSS J1307+2156 is well reproduced by $T_{\text {eff }}=8500 \pm 500 \mathrm{~K}$.

\section{LIGHT CURVE FITTING}

All light curves were fitted using a code written for the general case of binaries containing white dwarfs (see Copperwheat et al. 2010 for a detailed description). The program subdivides each star into small elements with a geometry fixed by its radius as measured along the direction of centres towards the other star. Roche geometry distortion and irradiation of the main-sequence star are included.

The basic parameters required to define the model are:

- $q=M_{\mathrm{MS}} / M_{\mathrm{WD}}$, the binary mass ratio

- $V_{s}=\left(K_{\mathrm{WD}}+K_{\mathrm{MS}}\right) / \sin i$, the sum of the unprojected stellar orbital speeds

- $i$, the binary inclination

- $R_{\mathrm{WD}} / a$ and $R_{\mathrm{MS}} / a$, the radii of the two stars scaled by the orbital separation, $a$.

- $T_{0}$, the time of mid-eclipse

- $P$, the binary period

- $T_{\text {eff,WD }}$ and $T_{\text {eff,MS }}$, the unirradiated temperatures of the two stars. Note that the temperatures are essentially just flux scaling parameters and only approximately correspond to the actual temperatures.

- $A$, the fraction of the irradiating flux from the white dwarf absorbed by the main-sequence star

- Gravity darkening coefficients for the main-sequence star

- Limb darkening coefficients for both stars

$q, V_{s}, i, R_{\mathrm{WD}} / a, R_{\mathrm{MS}} / a$ and $T_{0}$ were allowed to vary, although any spectroscopic constraints that we had for $V_{S}$ and $q$ were included in the fitting process. All data were phase-folded using previously published ephemerides, hence the period was kept fixed at a value of 1 . We kept $T_{\text {eff,WD }}$ fixed at the value determined from the spectroscopic fits and allowed $T_{\text {eff, MS }}$ to vary (we reiterate that these are only flux scaling parameters and so do not represent the true temperatures).

The irradiation is approximated by $\sigma T^{\prime 4}{ }_{\mathrm{MS}}=\sigma T_{\mathrm{MS}}^{4}+$ $A F_{\text {irr }}$ where $T^{\prime}$ MS is the modified temperature and $T_{\mathrm{MS}}$ is the temperature of the unirradiated main-sequence star, $\sigma$ is the Stefan-Boltzmann constant, $A$ is the fraction of the irradiating flux from the white dwarf absorbed by the mainsequence star and $F_{\text {irr }}$ is the irradiating flux, accounting for the angle of incidence and distance from the white dwarf. The value of $A$ makes little difference to the shape of the eclipse of the white dwarf, since at this phase the heated side of the main-sequence star is pointing away from Earth. Therefore, for most of our targets (those with just white dwarf eclipse data) this was fixed at a value of 0.5 . Otherwise it was allowed to vary freely.

The gravity darkening coefficients for the main-sequence stars were taken from Claret \& Bloemen (2011) for the appropriate filter. Note that this only has an effect in systems that are very close to Roche lobe filling. For both stars we adopted a four-parameter non-linear limb darkening model (see Claret 2000), given by

$\frac{I(\mu)}{I(1)}=1-\sum_{k=1}^{4} a_{k}\left(1-\mu^{\frac{k}{2}}\right)$,

where $\mu=\cos \phi(\phi$ is the angle between the line of sight and the emergent flux), and $I(1)$ is the monochromatic specific intensity at the centre of the stellar disk. Parameters for the 


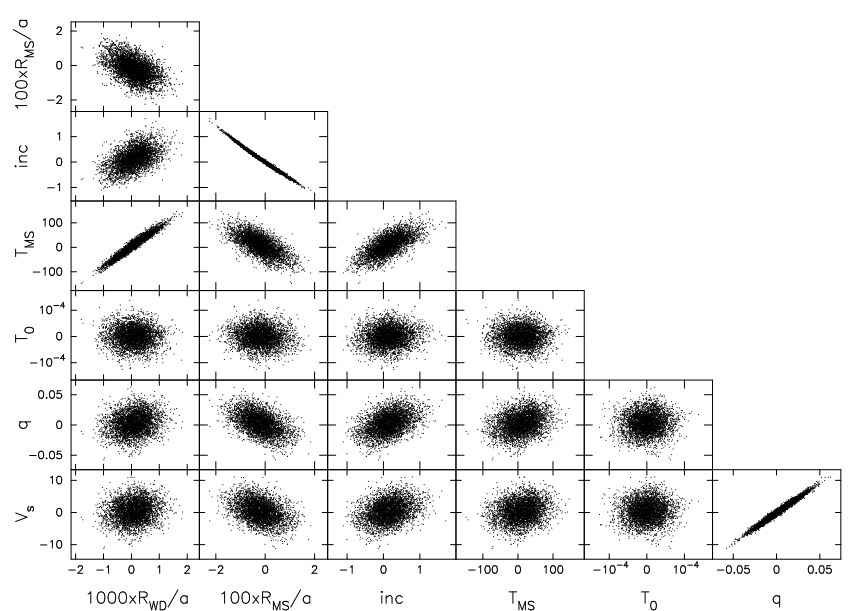

Figure 4. An example of the posterior probability distributions from one of our MCMC chains fitting the light curve of SDSS J0024+1745. In this case the probability was driven by a combination of the $\chi^{2}$ of the fit to the light curve and a prior probability from the spectroscopic mass ratio, radial velocity and rotational broadening measurements. The mean values of each parameter have been subtracted and only 10 per cent of the points are shown for clarity.

white dwarfs were taken from Gianninas et al. (2013) and from Claret \& Bloemen (2011) for the main-sequence stars and held fixed during the fitting process. While this means that our mass-radius measurements are not completely independent of model atmosphere calculations, the adopted values of the limb darkening coefficients have a very minor impact on the final physical parameters of the stars, typically at a level below their statistical uncertainties (Parsons et al. 2016).

We used the Markov Chain Monte Carlo (MCMC) method to determine the distributions of our model parameters (Press et al. 2007). The MCMC method involves making random jumps in the model parameters, with new models being accepted or rejected according to their probability computed as a Bayesian posterior probability. This probability is driven by a combination of the $\chi^{2}$ of the fit to the light curve data and any additional prior probabilities from our spectroscopic data, which allow us to implement the inclination and radii degeneracy breaking techniques outlined in Section 3. For each target an initial MCMC chain was used to determine the approximate best parameter values and covariances. These were then used as the starting values for longer chains which were used to determine the final model values and their uncertainties. Four chains were run simultaneously to ensure that they converged on the same values. The first 50,000 points from each chain were classified as a "burn-in" phase and were removed for the subsequent analysis, to ensure the final results were not skewed towards the initial values. An example of the typical parameter distributions is shown in Figure 4.

\section{RESULTS}

In this section we detail the results for each object individually. We reiterate that the focus of this paper is testing the mass-radius relation for white dwarfs and the results for the main-sequence stars will be presented in a forthcoming paper. Model fits to all the primary eclipse light curves are shown in Figure 5. Additionally, in Figure 6 we plot the mass-radius constraints for all our white dwarfs along with different mass-radius relationships corresponding to $\mathrm{C} / \mathrm{O}$ core models and He core models with thick and thin surface hydrogen envelopes. White dwarf models are taken from Fontaine et al. (2001) and Benvenuto \& Althaus (1999) $\left(T_{\text {eff }}>40,000 \mathrm{~K}\right)$ for $\mathrm{C} / \mathrm{O}$ core white dwarfs and from Panei et al. (2007) for He core white dwarfs. In the following sections we quote the mass-radius values and their uncertainties from the $1 \sigma$ contours in Figure 6 .

\subsection{CSS 080502}

CSS 080502 (SDSS J090812.04+060421.2 in SIMBAD) was discovered to be an eclipsing white dwarf plus M dwarf binary with a period of $3.6 \mathrm{~h}$ by Drake et al. (2009). It is a relatively bright $(V=17.1)$ system containing a $17,800 \mathrm{~K}$ white dwarf with an M3.5 companion. This binary is ideal for precise mass-radius measurements since the white dwarf's spectral features dominate blueward of $\mathrm{H} \alpha$ while the $\mathrm{M}$ star's features dominate redward from this. Irradiation of the main-sequence star is also minor. For this system we used the rotational broadening of the main-sequence star to help constrain the orbital inclination.

The fit shows that the white dwarf has a mass and radius of $0.476 \pm 0.004 \mathrm{M}_{\odot}$ and $0.0175 \pm 0.0003 \mathrm{R}_{\odot}$, placing it in the region where both $\mathrm{C} / \mathrm{O}$ and He core white dwarfs may exist. However, while the radius is consistent with $\mathrm{He}$ core models, it is substantially larger than $\mathrm{C} / \mathrm{O}$ core models predict and would be 6 per cent oversized if it has a $\mathrm{C} / \mathrm{O}$ core (see Figure 6). The radius is also more consistent with a thick surface hydrogen envelope $\left(M_{\mathrm{H}} / M_{\mathrm{WD}}=10^{-4}\right)$ rather than thinner models.

\section{$7.2 \quad$ CSS 09704}

CSS 09704 (SDSS J220823.66-011534.1 in SIMBAD) is a hot $(30,000 \mathrm{~K})$ white dwarf with a low-mass M6 companion in a $3.8 \mathrm{~h}$ binary discovered by Drake et al. (2010). The white dwarf completely dominates the optical flux and substantially irradiates its main-sequence companion star, to the extent that no absorption lines from the companion are visible in the X-shooter spectrum. However, several strong emission lines originating from this star are seen (caused by the irradiation) and were used to constrain its radial velocity semi-amplitude using Equation 6. These lines (in conjunction with the white dwarf's features) can also be used to measure the gravitational redshift of the white dwarf and hence constrain the physical parameters of the stars.

The mass and radius of the white dwarf were determined to be $0.416 \pm 0.036 \mathrm{M}_{\odot}$ and $0.0252 \pm 0.0017 \mathrm{R}_{\odot}$. Despite the relatively large uncertainties on these measurements, the radius is consistent with $\mathrm{He}$ core models, but substantially oversized for $\mathrm{C} / \mathrm{O}$ core models (by 25 per cent). The hydrogen layer mass cannot be constrained from our measurements. 

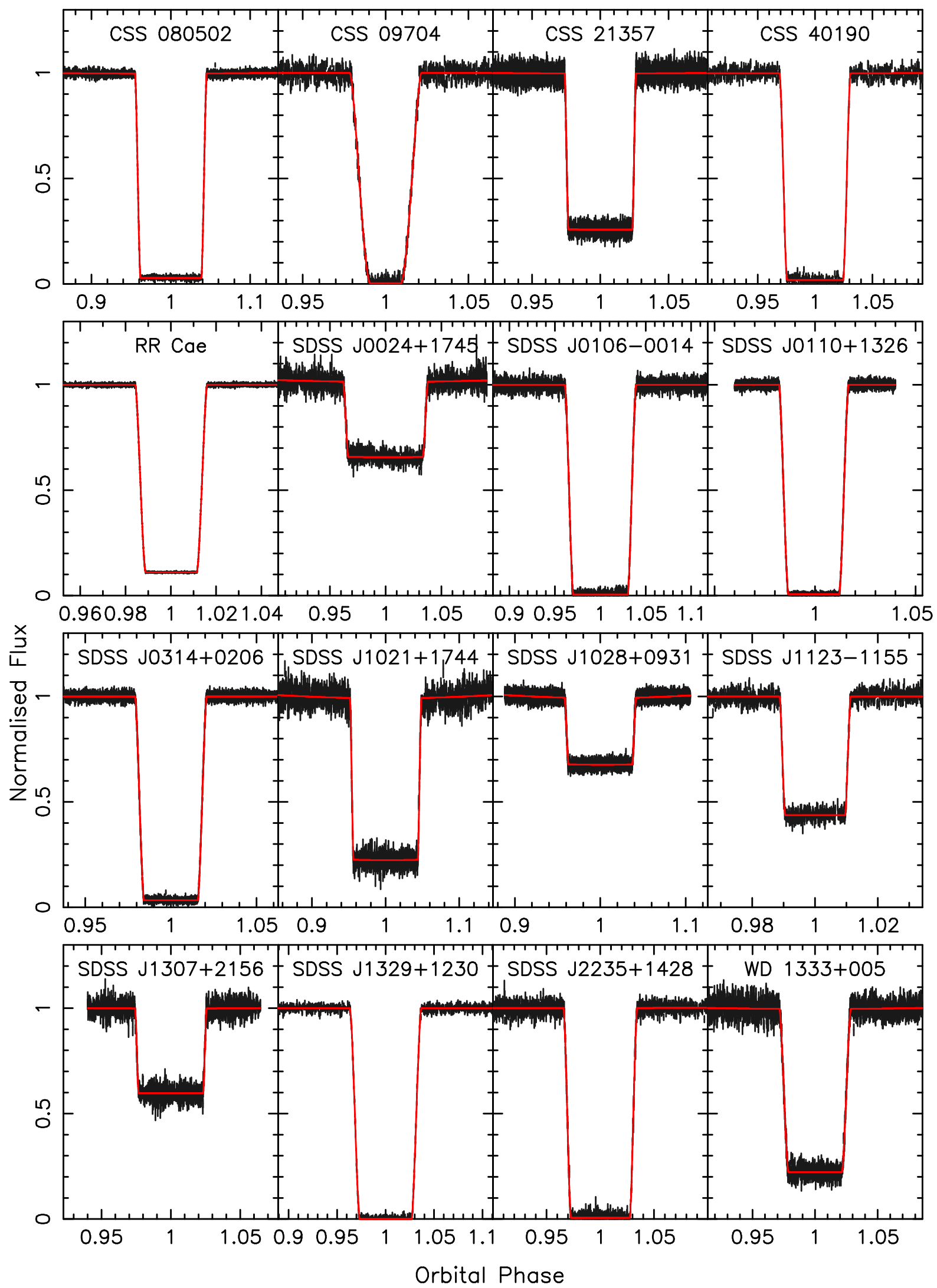

Figure 5. Light curves of the eclipse of the white dwarf in all our systems. The best fit models are shown in red. The light curves shown are in the $g^{\prime}$ band or the KG5 band (for CSS 21357, SDSS J1028+0931, SDSS J1123-1155, SDSS J1307+2156 and WD 1333+005). 

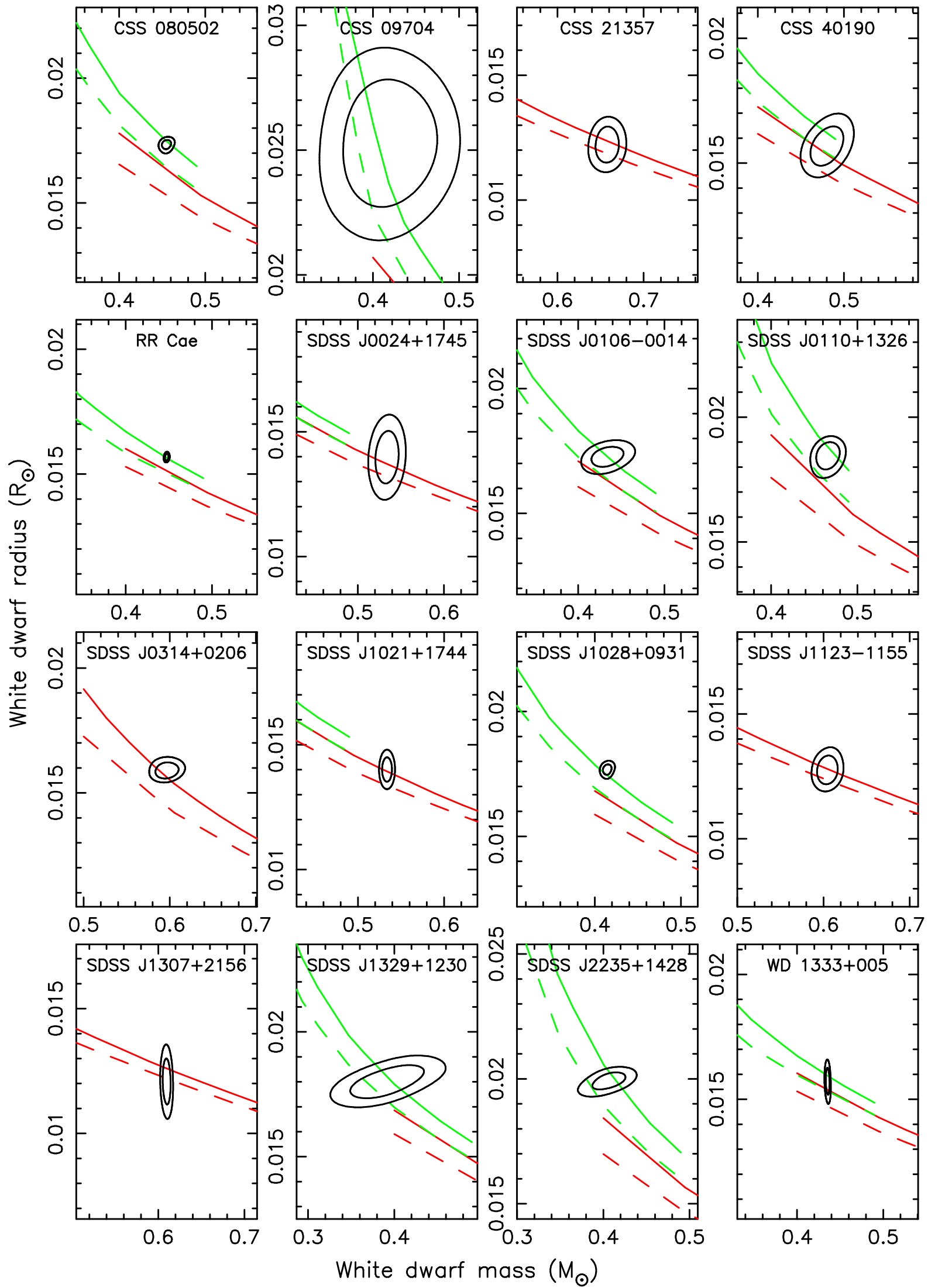

Figure 6. Constraints on the masses and radii of the white dwarfs in all our systems, shown as contours (68 and 95 percentile regions). $\mathrm{C} / \mathrm{O}$ core models for the measured white dwarf temperature are shown in red for thick $\left(\mathrm{solid}, M_{\mathrm{H}} / M_{\mathrm{WD}}=10^{-4}\right)$ and thin $(\mathrm{dashed}$, $M_{\mathrm{H}} / M_{\mathrm{WD}}=10^{-10}$ ) hydrogen envelopes (Fontaine et al. 2001) and He core models are shown in green for thick $\left(\right.$ solid, $M_{\mathrm{H}} / M_{\mathrm{WD}}=10^{-4}$ ) and thin (dashed, $M_{\mathrm{H}} / M_{\mathrm{WD}}=10^{-8}$ ) hydrogen envelopes (Panei et al. 2007). All plots are on the same scale and centred on the mean value for each white dwarf.

MNRAS 000, 1-19 (2017) 


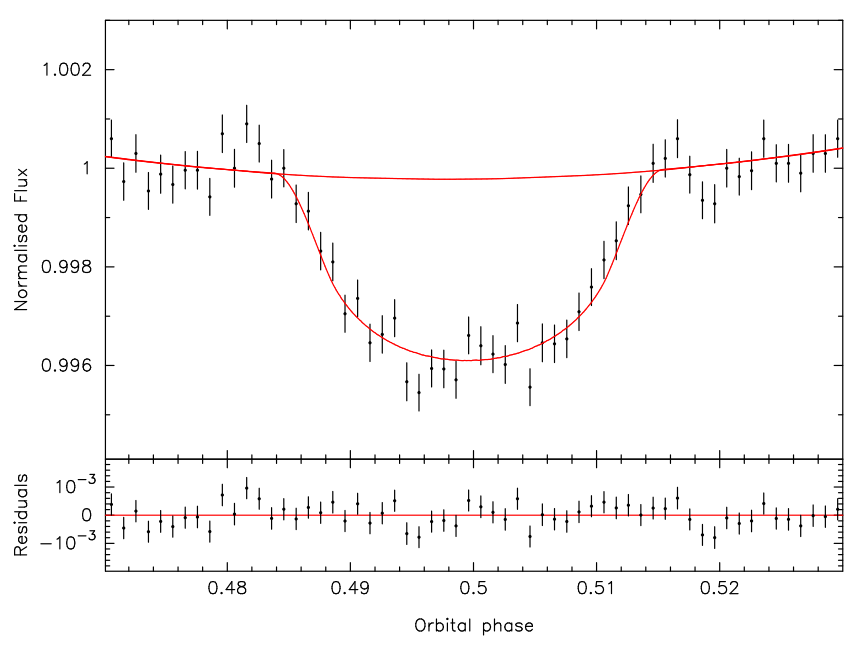

Figure 7. ULTRACAM $i^{\prime}$ band light curve of the secondary eclipse of RR Cae (i.e. the transit of the white dwarf across the face of the main-sequence star). The best fit model is shown in red as well as a model in which the secondary eclipse has been turned off, demonstrating its shallow depth and also the small amount of ellipsoidal modulation present (which causes the curve in the out-of-eclipse data)

\subsection{CSS 21357}

CSS 21357 (SDSS J134841.61+183410.5 in SIMBAD) was discovered by Drake et al. (2010) to be an eclipsing binary containing a 16,000 K white dwarf and an M3 main-sequence star in a $6.0 \mathrm{~h}$ binary. Clean features from both stars are visible in the X-shooter spectra and the rotational broadening of the $\mathrm{M}$ star was used to constrain the system inclination.

The white dwarf's mass and radius are $0.658 \pm 0.010 \mathrm{M}_{\odot}$ and $0.0122 \pm 0.0005 \mathrm{R}_{\odot}$. The radius is consistent with $\mathrm{C} / \mathrm{O}$ core models, though the uncertainty is too large to constrain the hydrogen layer mass.

\subsection{CSS 40190}

CSS 40190 (SDSS J083845.86+191416.5 in SIMBAD) is an eclipsing system consisting of a $14,900 \mathrm{~K}$ white dwarf and an M5 main-sequence star in a $3.1 \mathrm{~h}$ period discovered by Drake et al. (2010). While the white dwarf dominates the optical spectrum, features from the main-sequence star are still visible and the star is not strongly irradiated, therefore reliable velocities could be measured from both stars. Gravitational redshift and rotational broadening measurements were also made, with the broadening measurement used to constrain the inclination.

The resultant mass and radius measurements for the white dwarf were $0.482 \pm 0.008 \mathrm{M}_{\odot}$ and $0.0158 \pm 0.0004 \mathrm{R}_{\odot}$, placing it in a region where both $\mathrm{C} / \mathrm{O}$ and He core white dwarfs may reside. The measured radius is marginally more consistent with a $\mathrm{C} / \mathrm{O}$ core white dwarf, but a He core cannot be ruled out in this case, as shown in Figure 6 .

\subsection{RR Cae}

RR Cae is a bright $(V=14.4)$ close-by (20pc, Subasavage et al. 2009) cool 7,540 K DAZ white dwarf in a $7.3 \mathrm{~h}$ binary with an M4 main-sequence star, discovered to be eclipsing by Krzeminski (1984). We did not observe RR Cae spectroscopically with X-shooter because archival high quality UVES data already exist and were thoroughly analysed by Ribeiro et al. (2013). We use their spectroscopic constraints (listed in Table 1) in addition to our own light curve data to constrain the system parameters.

RR Cae is the only one of our systems in which the secondary eclipse is visible (though only in the $i^{\prime}$ band) and can be used to constrain the inclination and radii of both stars; note that several previously published systems also show secondary eclipses: NN Ser (Parsons et al. 2010) and SDSS J0857+0342 (Parsons et al. 2012a). In Figure 7 we show the secondary eclipse light curve, which is a combination of six separate observations. Additionally, the mainsequence star in RR Cae is particularly active and often flares, hence two observations had to be excluded due to flares interfering with the eclipse. Nevertheless, the secondary eclipse is well detected (despite its shallow $\sim 0.4$ per cent depth) and, in combination with the primary eclipse and spectroscopic data, places firm constraints on the mass and radius of the white dwarf of $0.448 \pm 0.002 \mathrm{M}_{\odot}$ and $0.01568 \pm 0.00009 R_{\odot}$. The measured radius is consistent with the predictions of He core white dwarf models with a thick surface hydrogen layer, but is slightly oversized for a $\mathrm{C} / \mathrm{O}$ core white dwarf.

\subsection{SDSS J0024+1745}

SDSS J0024+1745 (SDSS J002412.87+174531.4 in SIMBAD) was discovered as an eclipsing binary by Parsons et al. (2015). It shows the shallow eclipse of a cool 8,300 K white dwarf by an M2.5 main-sequence star in a $4.8 \mathrm{~h}$ orbit. The main-sequence star dominates the optical spectrum, but white dwarf features are visible in the X-shooter spectrum, specifically the narrow $\mathrm{Ca}$ II $\mathrm{H}$ and $\mathrm{K}$ lines, which yielded high precision velocity measurements. The rotational broadening of the M star was used to constrain the orbital inclination and physical parameters of the stars.

We determined the mass and radius of the white dwarf to be $0.534 \pm 0.009 \mathrm{M}_{\odot}$ and $0.0140 \pm 0.0007 \mathrm{R}_{\odot}$, consistent with $\mathrm{C} / \mathrm{O}$ core models, though not precise enough to constrain the hydrogen layer mass.

\subsection{SDSS J0106-0014}

SDSS J0106-0014 (SDSS J010623.01-001456.3 in SIMBAD) was discovered to be an eclipsing binary by Kleinman et al. (2004), who noted the discrepancy between its SDSS photometric and spectroscopic magnitudes, indicating that the system was at least partially in eclipse during the photometric observations. It consists of a $14,000 \mathrm{~K}$ white dwarf and a very low-mass $\mathrm{M}$ dwarf companion in a short $2.0 \mathrm{~h}$ period.

Our spectroscopic data revealed absorption lines from the main-sequence star that allowed us to trace its motion, but were too weak to give reliable rotational broadening measurements. Therefore, we used the gravitational redshift of the white dwarf to help constrain the binary inclination. The mass and radius of the white dwarf were determined to be $0.441 \pm 0.014 \mathrm{M}_{\odot}$ and $0.0175 \pm 0.0008 \mathrm{R}_{\odot}$, consistent with He core models (although not precise enough to constrain the hydrogen layer mass) and slightly oversized for 


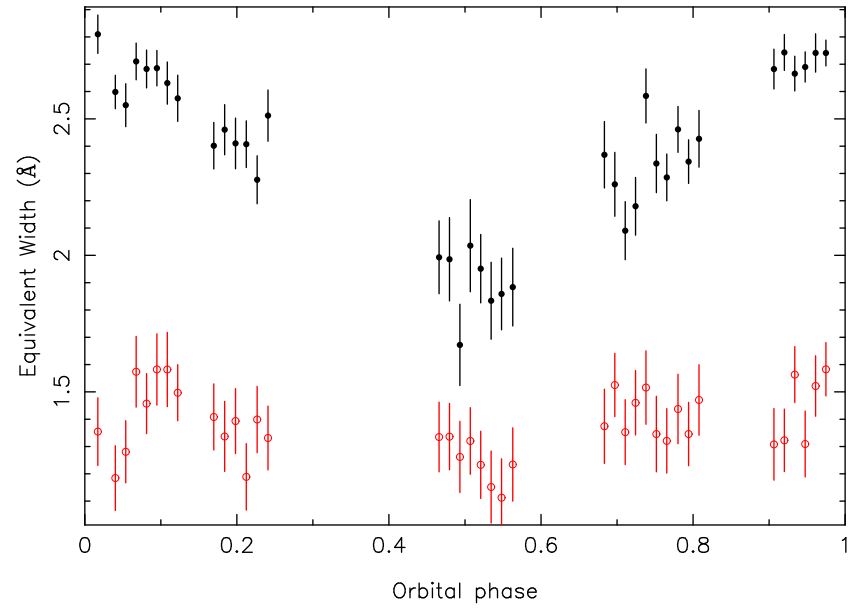

Figure 8. Measured equivalent width of the Na I $8183 / 94 \AA$ absorption doublet (filled black points) and the K I $12500 \AA$ absorption line (open red points) originating from the heated mainsequence star in SDSS J0110+1326. The sodium doublet clearly becomes weaker around phase 0.5, when the heated hemisphere of the star is visible, implying that these lines are affected by the irradiation from the white dwarf and hence do not reliably track the centre-of-mass of the main-sequence star. The potassium line shows no such variations in strength and therefore can be used to properly trace the motion of the star.

$\mathrm{C} / \mathrm{O}$ core models, although overlapping with thick hydrogen layer $\mathrm{C} / \mathrm{O}$ core models at the $2 \sigma$ level.

\subsection{SDSS J0110+1326}

SDSS J0110+1326 (SDSS J011009.08+132616.7 in SIM$\mathrm{BAD})$ is an eclipsing binary containing a relatively hot 24,500 K white dwarf and an M4.5 main-sequence star with a period of $8.0 \mathrm{~h}$ discovered by Pyrzas et al. (2009). The mainsequence star is moderately irradiated and therefore many of its absorption lines are actually slightly filled in at different orbital phases thus rendering them unreliable for tracking the centre-of-mass of the star. This was previously seen by Pyrzas et al. (2009) who noted the equivalent width of the main-sequence star's lines were highly phase dependent. However, our X-shooter data cover a much wider wavelength range than the Pyrzas et al. (2009) spectroscopy. Crucially, our data cover the near-infrared spectral range where several strong potassium lines are located. The K I $12500 \AA$ absorption line from the main-sequence star shows no variation in its equivalent width over the orbital period and therefore appears to be unaffected by the irradiating flux from the white dwarf (see Figure 8), allowing us to reliably trace its centre-of-mass as well as measure its rotational broadening. Note that the radial velocity measurement from the sodium doublet lines was $194.2 \pm 2.7 \mathrm{~km} \mathrm{~s}^{-1}$, slightly larger than the $190.9 \pm 1.2 \mathrm{~km} \mathrm{~s}^{-1}$ measured from the potassium line, demonstrating the distorting effect of irradiation.

The white dwarf's mass and radius are $0.466 \pm 0.009 \mathrm{M}_{\odot}$ and $0.0184 \pm 0.0004 \mathrm{R}_{\odot}$, consistent with $\mathrm{He}$ core models but slightly oversized for $\mathrm{C} / \mathrm{O}$ core models. The radius is also marginally more consistent with a thick hydrogen layer mass.

\subsection{SDSS J0314+0206}

SDSS J0314+0206 (WD 0312+019 in SIMBAD) was discovered by Drake et al. (2014) to be a deeply eclipsing binary containing a hot $46,800 \mathrm{~K}$ white dwarf with an M3 mainsequence star companion in a $7.3 \mathrm{~h}$ orbit. Despite the high temperature of the white dwarf, the main-sequence star's features are still visible in the X-shooter spectra, thanks to its relatively high mass. However, it is substantially irradiated by the white dwarf and there are numerous emission lines throughout the spectra and many of the absorption lines show variations in their strength at different orbital phases. Nevertheless, like SDSS J0110+1326, the K I $12500 \AA$ absorption line in the near-infrared shows no variations in strength throughout the orbit and therefore can be used to reliably track the motion of the main-sequence star. However, the absorption line is quite weak, since it is heavily diluted by the white dwarf's flux, to the extent that the rotational broadening measurement suffered from substantial uncertainty and hence would not strongly constrain the inclination, compared to other methods. Therefore, we relied on the gravitational redshift measurement to constrain the system parameters. As well as the standard Balmer absorption lines, the white dwarf spectrum also contains He II $4686 \AA$ and He I $5876 \AA$ absorption lines (hence this is a DAO white dwarf), the latter of which is relatively narrow allowing precise velocity measurements.

The white dwarf has a mass and radius of $0.596 \pm$ $0.009 \mathrm{M}_{\odot}$ and $0.0159 \pm 0.0002 \mathrm{R}_{\odot}$ and has a radius consistent with $\mathrm{C} / \mathrm{O}$ core models with a thick hydrogen envelope, as demonstrated in Figure 6.

\subsection{SDSS J1021+1744}

SDSS J1021+1744 (SDSS J102102.25+174439.9 in SIM$\mathrm{BAD})$ is a $10,600 \mathrm{~K}$ white dwarf in a $3.4 \mathrm{~h}$ binary with an M3 main-sequence companion discovered to eclipse by Parsons et al. (2013). Away from the eclipse, the light curve shows deep, sharp dips caused by prominence like structures originating from the $\mathrm{M}$ star that pass in front of the white dwarf (Irawati et al. 2016). We removed all the dips from each ULTRACAM light curve individually (by simply excluding any points obviously below the mean out-of-eclipse flux, plus a small buffer of 20 seconds either side of a dip), before combining them to make the final eclipse light curve. The three ULTRACAM observations of SDSS J1021+1744 were separated by more than four months and, as noted in Irawati et al. (2016), the dips vary in strength and position over long timescales, therefore, between the three observations we were able to construct a clean eclipse light curve.

While the M star's features are easily visible in the Xshooter spectra, the white dwarf's features are heavily diluted by the M star. However, the white dwarf accretes material from the M star's wind resulting in emission lines from the white dwarf in the Balmer series allowing us to track its motion (these emission features have been seen in other detached white dwarf plus main-sequence star binaries, e.g. Tappert et al. 2011; Parsons et al. 2012c). We used the rotational broadening measurement to constrain the system parameters.

The white dwarf has a mass and radius of $0.534 \pm$ $0.004 \mathrm{M}_{\odot}$ and $0.0140 \pm 0.0003 \mathrm{R}_{\odot}$, consistent with $\mathrm{C} / \mathrm{O}$ core 
models. The radius is also marginally more consistent with thick hydrogen layer models. Interestingly, our measurements place the white dwarf in SDSS J1021+1744 within the ZZ Ceti instability strip, although the uncertainty in temperature is large enough that it may still fall outside the strip. However, we see no evidence in our data for pulsations nor were there any obvious signs of pulsations in the long term monitoring data in Irawati et al. (2016).

\subsection{SDSS J1028+0931}

SDSS J1028+0931 (SDSS J102857.78+093129.8 in SIMBAD) is a binary containing a $12,200 \mathrm{~K}$ white dwarf and an M2.5 main-sequence star in a $5.6 \mathrm{~h}$ orbit discovered to eclipse by Parsons et al. (2013). The main-sequence star dominates the optical flux and hence the eclipse is shallow. However, white dwarf features are visible in the X-shooter spectra and, like SDSS J1021+1744, accretion of wind material drives emission lines in the Balmer series that were used to track the white dwarf's motion. We used the rotational broadening measurement to constrain the system parameters.

We determined the mass and radius of the white dwarf to be $0.415 \pm 0.004 \mathrm{M}_{\odot}$ and $0.0177 \pm 0.0002 \mathrm{R}_{\odot}$, consistent with He core models but oversized for $\mathrm{C} / \mathrm{O}$ models. The radius is also more consistent with thick hydrogen layer models than those with thinner layers. These parameters place the white dwarf close to the blue edge of the ZZ Ceti instability strip, but our (limited) photometry does not show any signs of pulsations.

\subsection{SDSS J1123-1155}

Discovered to be an eclipsing binary by Parsons et al. (2015)， SDSS J1123-1155 (SDSS J112308.40-115559.3 in SIMBAD) consists of a $10,200 \mathrm{~K}$ white dwarf in an $18.5 \mathrm{~h}$ orbit with a M3.5 main-sequence star, making it the longest period binary in our sample. Features from both stars are visible in the spectrum including narrow metal absorption lines in the white dwarf's spectrum caused by accreting wind material from the main-sequence star. However, due to the much longer period of this system, compared to the others in our sample, the rotational broadening of the $\mathrm{M}$ star is relatively small and in fact cannot be measured with the resolution of the $\mathrm{X}$-shooter data. Therefore, we rely on the gravitational redshift measurement to determine the inclination and hence physical parameters of the stars.

The white dwarf in SDSS J1123-1155 was found to have a mass and radius of $0.605 \pm 0.008 \mathrm{M}_{\odot}$ and $0.0128 \pm 0.0004 \mathrm{R}_{\odot}$, consistent with $\mathrm{C} / \mathrm{O}$ core models. However, the measurements are not precise enough to constrain the hydrogen layer mass.

\subsection{SDSS J1307+2156}

SDSS J1307+2156 (SDSS J130733.49+215636.7 in SIM$\mathrm{BAD}$ ) contains a cool $8,500 \mathrm{~K}$ white dwarf in a $5.2 \mathrm{~h}$ binary with an M4 main-sequence star and was discovered to be eclipsing by Parsons et al. (2013). Originally listed as a DC white dwarf from its SDSS spectrum (Rebassa-Mansergas et al. 2012), our higher resolution Xshooter data revealed narrow Balmer absorption lines as well as a large number of metal lines caused by wind accretion. The M star dominates the flux redward of $\mathrm{H} \beta$, hence we use the measurement of its rotational broadening to constrain the inclination and physical parameters of the binary.

We determined the mass and radius of the white dwarf to be $0.610 \pm 0.003 \mathrm{M}_{\odot}$ and $0.0121 \pm 0.0006 \mathrm{R}_{\odot}$, consistent with $\mathrm{C} / \mathrm{O}$ core models, though not precise enough to constrain the hydrogen layer mass.

\subsection{SDSS J1329+1230}

SDSS J1329+1230 (SDSS J132925.21+123025.4 in SIMBAD) was found to be an eclipsing binary by Drake et al. (2010). It contains a $12,500 \mathrm{~K}$ white dwarf with a low-mass M8 companion with a short $1.9 \mathrm{~h}$ period. The white dwarf completely dominates the optical spectrum and narrow calcium and magnesium absorption lines are visible in its spectrum due to it accreting some material from the wind of its companion. No absorption features are visible from the $\mathrm{M}$ star, though several emission lines are seen, arising from the heating effect from the white dwarf. We use these and Equation 6 to determine its radial velocity and use the gravitational redshift of the white dwarf to constrain the inclination and hence physical parameters of the stars.

The mass and radius of the white dwarf were found to be $0.392 \pm 0.023 \mathrm{M}_{\odot}$ and $0.0180 \pm 0.0005 \mathrm{R}_{\odot}$, making this the lowest mass white dwarf in our sample. The radius is consistent with He core models, but slightly too large for $\mathrm{C} / \mathrm{O}$ core models (4 per cent oversized, see Figure 6 ). The precision of the measurements is insufficient to constrain the hydrogen layer mass.

\subsection{SDSS J2235+1428}

SDSS J2235+1428 (SDSS J223530.62+142855.1 in SIMBAD) contains a $20,800 \mathrm{~K}$ white dwarf in a $3.5 \mathrm{~h}$ binary with an M5 main-sequence star and was discovered to be eclipsing by Parsons et al. (2013). The white dwarf dominates the optical spectrum, although features from the M star are visible redward of $\mathrm{H} \alpha$. The M star is moderately heated and several emission lines are visible throughout the spectrum. However, the sodium $8200 \AA$ absorption doublet appears to be unaffected by the heating, since the equivalent widths of the two lines show no variation with orbital phase. Therefore, we use these to track the main-sequence star's motion and measure its rotational broadening in order to constrain the inclination.

The white dwarf has a mass and radius of $0.398 \pm$ $0.022 \mathrm{M}_{\odot}$ and $0.0198 \pm 0.0005 \mathrm{R}_{\odot}$, consistent with He core models, but substantially oversized for a $\mathrm{C} / \mathrm{O}$ core white dwarf ( 8 per cent too large). The radius measurement is slightly more consistent with a thick hydrogen layer, but a thinner layer cannot be excluded.

\subsection{WD $1333+005$}

WD $1333+005$ was found to be an eclipsing binary by Drake et al. (2010). It contains a cool 7,700 K white dwarf and M5 main-sequence star with a $2.9 \mathrm{~h}$ period. The white dwarf's spectrum contains a huge number of narrow metal absorption lines due to accreting material from the wind 
Table 2. White dwarf mass-radius measurements obtained from eclipsing binaries. $R / R_{\mathrm{C} / \mathrm{O}}$ is the ratio of the measured radius to the theoretical radius of a $\mathrm{C} / \mathrm{O}$ core white dwarf with a thick $\left(M_{\mathrm{H}} / M_{\mathrm{WD}}=10^{-4}\right)$ hydrogen envelope (taken from Fontaine et al. 2001 or Benvenuto \& Althaus 1999 for $\left.T_{\text {eff }}>40,000 \mathrm{~K}\right), R / R_{\mathrm{He}}$ is the ratio of the measured radius to the theoretical radius of a He core white dwarf with a thick $\left(M_{\mathrm{H}} / M_{\mathrm{WD}}=10^{-4}\right)$ hydrogen envelope (Panei et al. 2007), for white dwarfs with masses less than $0.5 \mathrm{M}_{\odot}$. References: (1) This paper, (2) Bours et al. (2014), (3) Parsons et al. (2012b), (4) Parsons et al. (2010), (5) Parsons et al. (2016), (6) Parsons et al. (2012c), (7) Parsons et al. (2012a), (8) Pyrzas et al. (2012), (9) O'Brien et al. (2001).

\begin{tabular}{|c|c|c|c|c|c|c|c|c|}
\hline Object & $g$ mag & $\mathrm{P}_{\text {orb }}(\mathrm{h})$ & $T_{\mathrm{eff}}(\mathrm{K})$ & Mass $\left(\mathrm{M}_{\odot}\right)$ & Radius $\left(\mathrm{R}_{\odot}\right)$ & $R / R_{\mathrm{C} / \mathrm{O}}$ & $R / R_{\mathrm{He}}$ & Ref \\
\hline CSS 080502 & 17.08 & 3.587 & $17838 \pm 482$ & $0.4756 \pm 0.0036$ & $0.01749 \pm 0.00028$ & $1.06 \pm 0.01$ & $0.98 \pm 0.01$ & 1 \\
\hline CSS 09704 & 18.41 & 3.756 & $29969 \pm 679$ & $0.4164 \pm 0.0356$ & $0.02521 \pm 0.00170$ & $1.25 \pm 0.08$ & $1.05 \pm 0.08$ & 1 \\
\hline CSS 21357 & 17.29 & 5.962 & $15909 \pm 285$ & $0.6579 \pm 0.0097$ & $0.01221 \pm 0.00046$ & $0.98 \pm 0.03$ & - & 1 \\
\hline CSS 40190 & 18.16 & 3.123 & $14901 \pm 731$ & $0.4817 \pm 0.0077$ & $0.01578 \pm 0.00039$ & $1.02 \pm 0.03$ & $0.95 \pm 0.03$ & 1 \\
\hline CSS 41177A & 17.27 & 2.784 & $22497 \pm 60$ & $0.3780 \pm 0.0230$ & $0.02224 \pm 0.00041$ & $1.09 \pm 0.02$ & $0.98 \pm 0.02$ & 2 \\
\hline CSS 41177B & 17.27 & 2.784 & $11864 \pm 281$ & $0.3160 \pm 0.0110$ & $0.02066 \pm 0.00042$ & $1.05 \pm 0.02$ & $0.97 \pm 0.02$ & 2 \\
\hline GK Vir & 16.81 & 8.264 & $50000 \pm 673$ & $0.5618 \pm 0.0142$ & $0.01700 \pm 0.00030$ & $0.96 \pm 0.01$ & - & 3 \\
\hline NNSer & 16.43 & 3.122 & $63000 \pm 3000$ & $0.5354 \pm 0.0117$ & $0.02080 \pm 0.00020$ & $1.00 \pm 0.01$ & - & 4 \\
\hline QS Vir & 14.66 & 3.618 & $14220 \pm 350$ & $0.7816 \pm 0.0130$ & $0.01068 \pm 0.00007$ & $1.00 \pm 0.01$ & - & 5 \\
\hline RR Cae & 14.57 & 7.289 & $7540 \pm 175$ & $0.4475 \pm 0.0015$ & $0.01568 \pm 0.00009$ & $1.03 \pm 0.01$ & $1.00 \pm 0.01$ & 1 \\
\hline SDSS J0024+1745 & 18.71 & 4.801 & $8272 \pm 580$ & $0.5340 \pm 0.0090$ & $0.01398 \pm 0.00070$ & $1.01 \pm 0.05$ & - & 1 \\
\hline SDSS J0106-0014 & 18.14 & 2.040 & $13957 \pm 531$ & $0.4406 \pm 0.0144$ & $0.01747 \pm 0.00083$ & $1.05 \pm 0.01$ & $1.00 \pm 0.01$ & 1 \\
\hline SDSS J0110+1326 & 16.53 & 7.984 & $24569 \pm 385$ & $0.4656 \pm 0.0091$ & $0.01840 \pm 0.00036$ & $1.07 \pm 0.02$ & $0.99 \pm 0.02$ & 1 \\
\hline SDSS J0138-0016 & 18.84 & 1.746 & $3570 \pm 100$ & $0.5290 \pm 0.0100$ & $0.01310 \pm 0.00030$ & $0.99 \pm 0.02$ & - & 6 \\
\hline SDSS J0314+0206 & 16.95 & 7.327 & $46783 \pm 7706$ & $0.5964 \pm 0.0088$ & $0.01594 \pm 0.00022$ & $1.01 \pm 0.01$ & - & 1 \\
\hline SDSS J0857+0342 & 17.95 & 1.562 & $37400 \pm 400$ & $0.5140 \pm 0.0490$ & $0.02470 \pm 0.00080$ & $1.43 \pm 0.06$ & $1.08 \pm 0.06$ & 7 \\
\hline SDSS J1021+1744 & 19.51 & 3.369 & $10644 \pm 1721$ & $0.5338 \pm 0.0038$ & $0.01401 \pm 0.00032$ & $1.00 \pm 0.02$ & - & 1 \\
\hline SDSS J1028+0931 & 16.40 & 5.641 & $12221 \pm 765$ & $0.4146 \pm 0.0036$ & $0.01768 \pm 0.00020$ & $1.07 \pm 0.01$ & $1.02 \pm 0.01$ & 1 \\
\hline SDSS J1123-1155 & 17.99 & 18.459 & $10210 \pm 87$ & $0.6050 \pm 0.0079$ & $0.01278 \pm 0.00037$ & $0.99 \pm 0.02$ & - & 1 \\
\hline SDSS J1210+3347 & 16.94 & 2.988 & $6000 \pm 200$ & $0.4150 \pm 0.0100$ & $0.01590 \pm 0.00050$ & $1.02 \pm 0.03$ & $0.99 \pm 0.03$ & 8 \\
\hline SDSS J1212-0123 & 16.77 & 8.061 & $17707 \pm 35$ & $0.4393 \pm 0.0022$ & $0.01680 \pm 0.00030$ & $1.00 \pm 0.01$ & $0.94 \pm 0.01$ & 3 \\
\hline SDSS J1307+2156 & 18.25 & 5.192 & $8500 \pm 500$ & $0.6098 \pm 0.0031$ & $0.01207 \pm 0.00061$ & $0.96 \pm 0.04$ & - & 1 \\
\hline SDSS J1329+1230 & 17.26 & 1.943 & $12491 \pm 312$ & $0.3916 \pm 0.0234$ & $0.01800 \pm 0.00052$ & $1.04 \pm 0.02$ & $0.99 \pm 0.02$ & 1 \\
\hline SDSS J2235+1428 & 18.59 & 3.467 & $20837 \pm 773$ & $0.3977 \pm 0.0220$ & $0.01975 \pm 0.00050$ & $1.08 \pm 0.02$ & $0.98 \pm 0.02$ & 1 \\
\hline V471 Tau & 10.04 & 12.508 & $34500 \pm 1000$ & $0.8400 \pm 0.0500$ & $0.01070 \pm 0.00070$ & $1.06 \pm 0.06$ & - & 9 \\
\hline WD $1333+005$ & 17.41 & 2.927 & $7740 \pm 73$ & $0.4356 \pm 0.0016$ & $0.01570 \pm 0.00036$ & $1.02 \pm 0.02$ & $0.98 \pm 0.02$ & 1 \\
\hline
\end{tabular}

of the main-sequence star. The $\mathrm{M}$ star dominates the flux beyond $\mathrm{H} \beta$ and therefore we use the measurement of its rotational broadening to constrain the system parameters.

The mass and radius of the white dwarf are $0.436 \pm$ $0.002 \mathrm{M}_{\odot}$ and $0.0157 \pm 0.0004 \mathrm{R}_{\odot}$. Despite the high precision of our measurements, the radius is in agreement with both $\mathrm{He}$ and $\mathrm{C} / \mathrm{O}$ core models (since the difference between the models is small at lower temperatures), although the measured radius is inconsistent with thin hydrogen layer $\mathrm{C} / \mathrm{O}$ core models.

\section{DISCUSSION}

We summarise all our measurements in Table 2 along with other, previously published precise white dwarf mass-radius measurements from close binaries. We use the term "precise" to mean any mass and radius measurements that have been made independent of any theoretical mass-radius relationships or spectral fits and with uncertainties less than 10 per cent.

The white dwarfs in Table 2 span a wide range of masses and temperatures, as is best seen in Figure 9, although there is a clear lack of massive $\left(>1 \mathrm{M}_{\odot}\right)$ white dwarfs, which is unsurprising given the evolutionary history of these binaries. Note that these are currently the only white dwarfs with independent mass and radius measurements, since white dwarfs in astrometric binaries or common proper motion systems still rely on spectral fits to determine their emergent flux and hence radii. While Figure 9 nicely demonstrates the inverse mass-radius relationship for white dwarfs (i.e. that more massive white dwarfs are generally smaller than less massive ones, with some dependence upon temperature), it is not straightforward to assess the agreement with evolutionary models due to the different temperatures and core compositions of all these white dwarfs. Therefore, in Figure 10 we plot the ratio of the measured radius to the theoretical radius (taking into account the uncertainty in temperature as well), assuming thick surface hydrogen layers. For white dwarfs with masses less than $0.5 \mathrm{M}_{\odot}$ we plot this ratio relative to $\mathrm{C} / \mathrm{O}$ core models (in black) and He core models (in red). Both Figure 6 and Figure 10 show that white dwarfs with masses below $0.5 \mathrm{M}_{\odot}$ have radii that are more consistent with He core models and only one object, SDSS J1212-0123, has a radius more consistent with a C/O core (Parsons et al. 2012b). It is perhaps unsurprising that these low mass white dwarfs likely have He cores since, although it is possible to create $\mathrm{C} / \mathrm{O}$ core white dwarfs with masses less than $0.5 \mathrm{M}_{\odot}$, it requires substantial mass loss along the red giant branch (Prada Moroni \& Straniero 2009; Willems \& Kolb 2004; Han et al. 2000). For this to occur in a binary system requires that the initial mass ratio was large and therefore the orbit should increase during the mass loss and hence we would not observe such systems as close binaries. Nevertheless, this is the first direct observational evidence of a change in core composition in this 

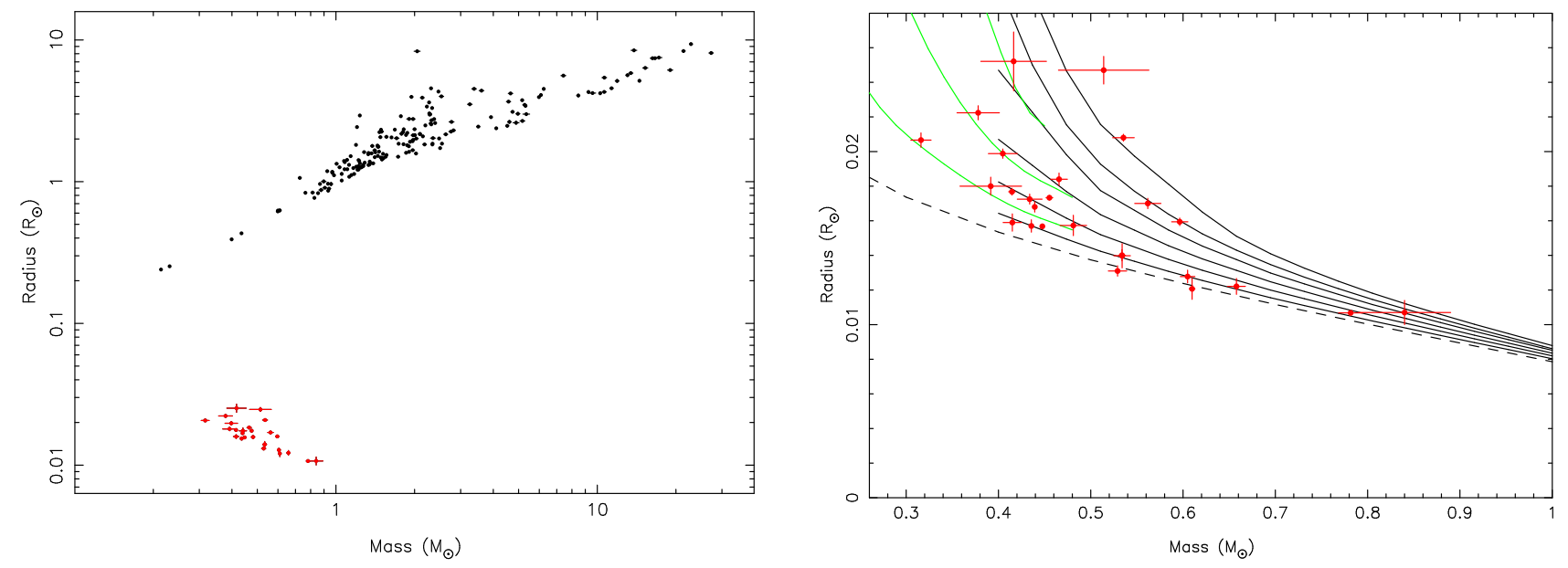

Figure 9. Left: stars and white dwarfs with precise model-independent mass and radius measurements. The white dwarfs are shown in red and are from Table 2, the main-sequence stars are taken from Torres et al. (2010). This figure demonstrates not only the inverse mass-radius relationship for white dwarfs, but also the large mass losses involved in stellar evolution. Right: The white dwarf mass-radius relationship. The solid black lines are theoretical C/O core models with thick hydrogen layers with temperatures from 60,000 K (top) to $10,000 \mathrm{~K}$ (bottom) in steps of 10,000 K (taken from Fontaine et al. 2001 or Benvenuto \& Althaus 1999 for $T_{\text {eff }}>40,000 \mathrm{~K}$ ). The green lines are He core models with thick hydrogen layers with temperatures from $30,000 \mathrm{~K}$ (top) to 10,000 K (bottom) in steps of $10,000 \mathrm{~K}$ (taken from Panei et al. 2007). The dashed black line is the zero-temperature relation from Verbunt \& Rappaport (1988).

mass range. With a sufficiently large sample of mass-radius measurements in this range one could even pinpoint exactly where this core change occurs (although this also depends upon the metallicity and so will not be the same for every object).

In addition to mass, temperature and core composition, the radius of a white dwarf is also dependent upon the surface hydrogen layer. Studies of pulsating ZZ Ceti type white dwarfs have found a large spread in the hydrogen layer mass of white dwarfs, from as low as $M_{\mathrm{H}} / M_{\mathrm{WD}}=$ $10^{-10}$ upto $M_{\mathrm{H}} / M_{\mathrm{WD}}=10^{-4}$ (Castanheira \& Kepler 2009; Romero et al. 2012), with a mean value of $M_{\mathrm{H}} / M_{\mathrm{WD}}=$ $2.71 \times 10^{-5}$. While our measurements cannot constrain the layer mass as precisely as pulsational studies, we can still potentially differentiate between thick and thin layers. In Figure 11 we plot the ratio of the measured white dwarf radius to the theoretical radius (assuming He cores for masses below $\left.0.5 \mathrm{M}_{\odot}\right)$ for thick hydrogen layers $\left(M_{\mathrm{H}} / M_{\mathrm{WD}}=10^{-4}\right.$, black points) and thin hydrogen layers $\left(M_{\mathrm{H}} / M_{\mathrm{WD}}=10^{-10}\right.$, red points). For objects with precise enough measurements we find that models with thick hydrogen layers reproduce the observed radii far better than models with thinner layers (that are often around 10 per cent too small). This effect is largest in hotter white dwarfs, where the layer mass has a much larger impact on the overall radius of the white dwarf.

We also compared our measured radii to the models of Benvenuto \& Althaus (1999) (C/O core) and Panei et al. (2007) (He core) over a range of different hydrogen layer masses (note that the Fontaine et al. 2001 models used in Figure 11 are only available with thick, $M_{\mathrm{H}} / M_{\mathrm{WD}}=10^{-4}$, or thin, $M_{\mathrm{H}} / M_{\mathrm{WD}}=10^{-10}$ layers). Model radii within one sigma of the measured value were deemed consistent (taking into account the uncertainties in the mass and temperature of each white dwarf) and are shown in Figure 12. We find that, for white dwarfs with precise enough measurements, their radii are consistent with having surface hydro-

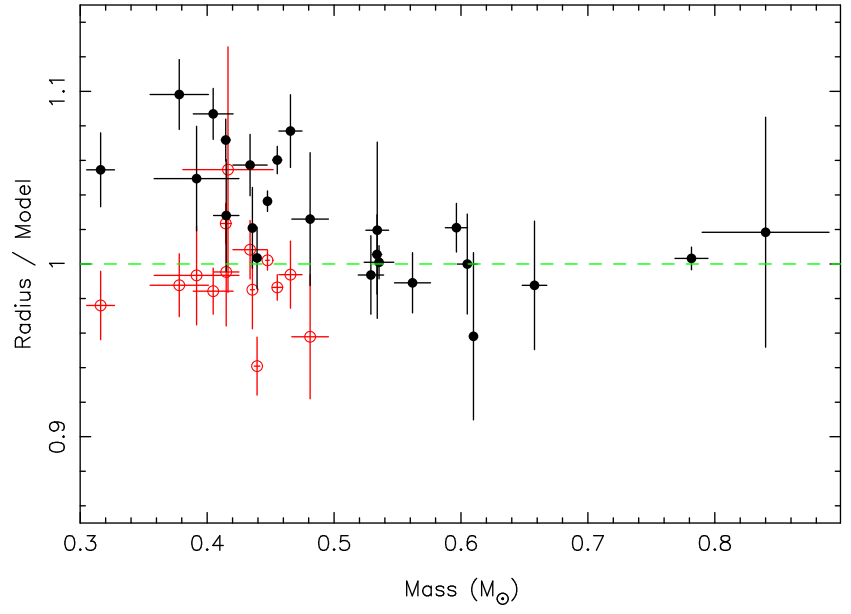

Figure 10. Ratio of the measured white dwarf radii to theoretical predictions as a function of mass. Filled black points are assuming a $\mathrm{C} / \mathrm{O}$ core white dwarf with a thick hydrogen layer (taken from Fontaine et al. 2001 or Benvenuto \& Althaus 1999 for $T_{\text {eff }}>40,000 \mathrm{~K}$ ), while open red points are assuming a He core with a thick hydrogen layer (for the same white dwarfs, with masses below $0.5 \mathrm{M}_{\odot}$, taken from Panei et al. 2007). Virtually every radius measurement below $0.5 \mathrm{M}_{\odot}$ is more consistent with $\mathrm{He}$ core rather than $\mathrm{C} / \mathrm{O}$ core models, the only exception being SDSS J1212-0123 (Parsons et al. 2012b).

gen layers with masses between $10^{-5} \geq M_{\mathrm{H}} / M_{\mathrm{WD}} \geq 10^{-4}$, with some spread to larger and smaller values. This is consistent with the canonical value of $10^{-4}$ from evolutionary computations (e.g. Althaus et al. 2010). Since all these white dwarfs have interacted with their main-sequence companions in the past (during the giant phase, through a common envelope event) it might be expected that they posses thinner hydrogen envelopes than isolated white dwarfs, although the small amount of material accreted from the wind 


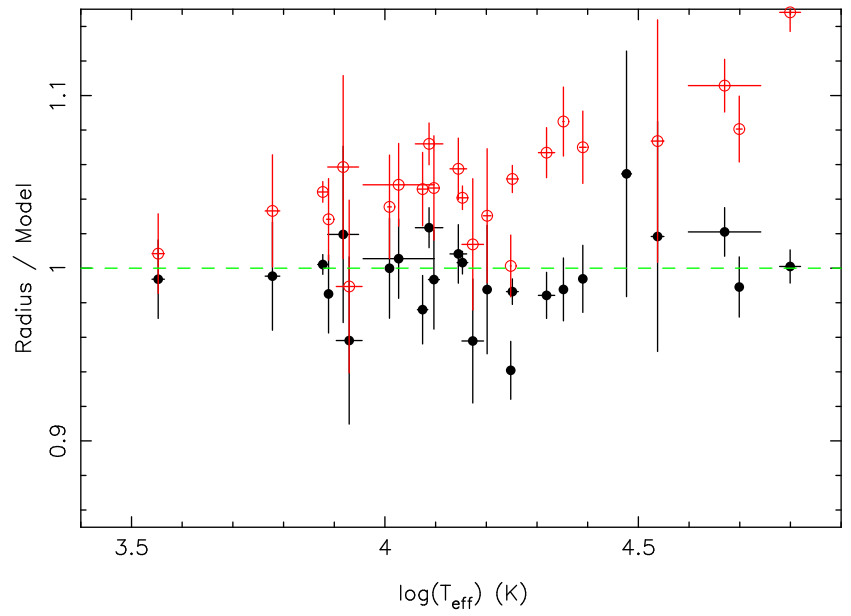

Figure 11. Ratio of the measured white dwarf radii to theoretical predictions as a function of effective temperature. Filled black points assume a thick surface hydrogen layer, while open red points assume a thin layer. The discrepancy is largest for hotter white dwarfs, with the majority of measurements favoring a thick layer.

of their companions $\left(\sim 10^{-15} \mathrm{M}_{\odot} \mathrm{yr}^{-1}\right.$ Pyrzas et al. 2012) ensures that there are no DB white dwarfs in close binaries with main-sequence stars (Parsons et al. 2013). However, the fact that our measurements are consistent with the canonical value implies that the hydrogen envelopes are not strongly affected by common envelope evolution. On the other hand, the hydrogen layer mass of the ZZ Ceti white dwarf in the close binary SDSS J113655.17+040952.6 was found to be $M_{\mathrm{H}} / M_{\mathrm{WD}} \approx 10^{-4.9}$ (Hermes et al. 2015), at the lower end of the range we find and potentially indicative of some small mass loss. A more robust test of whether or not the common envelope phase has an effect on the layer mass will require the identification of more $\mathrm{ZZ} \mathrm{Ceti} \mathrm{white} \mathrm{dwarfs}$ in close binaries.

\subsection{The accuracy of white dwarf spectroscopic fits}

For the vast majority of single white dwarfs, spectroscopic fits to the Balmer lines are currently the only method to estimate their masses (using the surface gravity determined from the fits and a mass-radius relation), and most of our knowledge of the white dwarf mass distribution is based on this method (e.g. Bergeron et al. 1992; Finley et al. 1997; Kepler et al. 2007; Tremblay et al. 2013). Our sample of white dwarfs in eclipsing binaries provides an excellent opportunity to test the precision and robustness of the spectrosopic fits, as we can compare the surface gravities derived from the X-Shooter spectra with those computed from the masses and radii that we directly measured from the light curve fits.

Figure 13 shows the comparison between the surface gravities of our white dwarfs from the spectrosopic fits and computed from the mass and radius values measured from the light curves. In general there is excellent agreement between the spectroscopic surface gravities and the measured values, however, there are a few outliers and in all these cases the spectroscopic fit over-predicts the surface gravity. Three

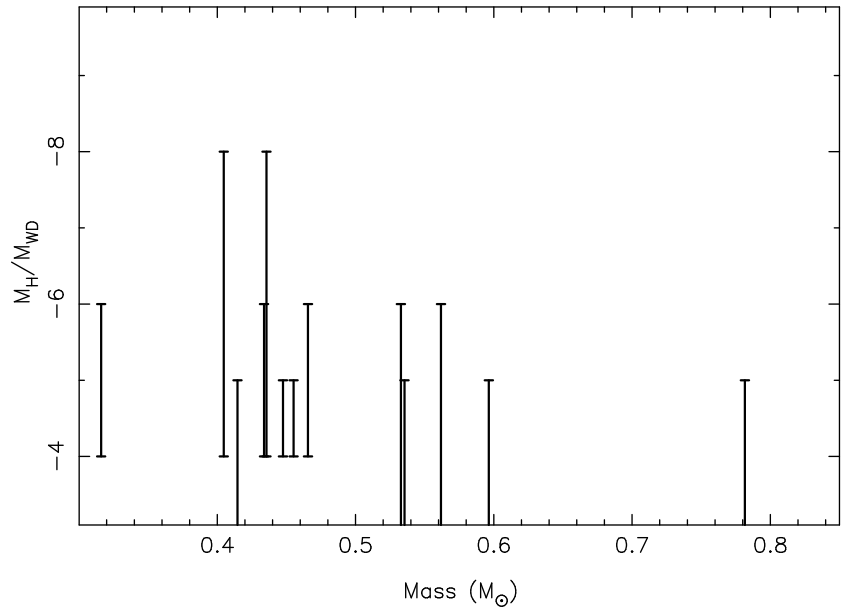

Figure 12. Range of surface hydrogen layer masses consistent with our measured radii. We have excluded objects where we have minimal or no constraints.

of these objects (SDSS J0024+1745, SDSS J1021+1744 and SDSS J1028+0931) are cool white dwarfs with early M-type companions that dominate the optical flux over the white dwarf. Therefore, the white dwarf spectra are of lower quality than the other objects and may still be contaminated by features from the main-sequence stars (as noted in Section 5.1, the main-sequence star subtraction is never perfect). This means that the spectroscopic fits are less reliable, since the Balmer lines may be distorted and hence it is perhaps unsuprising that these particular objects are outliers.

The one other outlier is SDSS J1123-1155, which differs from the other systems because the white dwarf dominates the optical light and therefore suffers from less contamination than the other objects. However, in this case the discrepancy is only at the 2 sigma level (spectroscopic $\log g=8.31 \pm 0.14$, eclipse analysis $\log g=8.01 \pm 0.03)$. As the model atmosphere fit measures the surface gravity from the width and shape of the Balmer line profiles, additional broadening mechanisms such as additional perturbers contributing the the Stark broadening (e.g. neutral He), or a magnetic field, may be the cause for the slightly higher spectroscopically determined mass.

\section{CONCLUSIONS}

We have used a combination of high-speed photometry and phase-resolved spectroscopy to precisely measure the masses and radii for 16 white dwarfs in detached eclipsing binaries with low-mass main-sequence star companions. We combined these results with 10 previously measured binaries to test the white dwarf mass-radius relationship. We found excellent agreement between our measured radii and theoretical predictions across a wide range of masses and temperatures. We also find that, as expected, the radii of white dwarfs with masses below $0.5 \mathrm{M}_{\odot}$ are far more consistent with $\mathrm{He}$ core models than those with $\mathrm{C} / \mathrm{O}$ cores. Moreover, our most precise measurements allow us to exclude thin surface hydrogen layers, with thicker values of $10^{-5} \geq M_{\mathrm{H}} / M_{\mathrm{WD}} \geq 10^{-4}$ favoured, implying that surface 


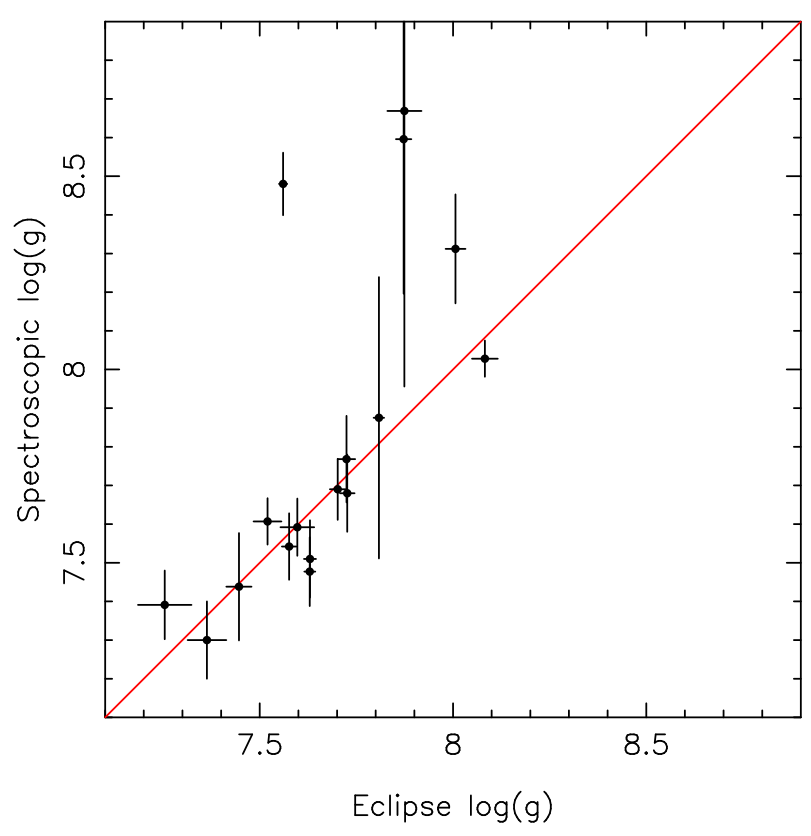

Figure 13. Comparison between the surface gravities determined from the model atmosphere fits to the X-Shooter spectroscopy, and those computed from the mass and radius values measured from the light curves.

hydrogen layers are not strongly depleted by close binary evolution.

\section{ACKNOWLEDGEMENTS}

We thank the referee for useful comments and suggestions. SGP acknowledges the support of the Leverhulme Trust. The research leading to these results has received funding from the European Research Council under the European Union's Seventh Framework Programme (FP/20072013) / ERC Grant Agreement numbers 340040 (HiPERCAM) and 320964 (WDTracer). ULTRACAM, TRM, VSD, SPL and EB are supported by the Science and Technology Facilities Council (STFC). MCPB acknowledges support from the Joint Committee ESO-Government of Chile (grant 2014). ARM acknowledges financial support from MINECO grant AYA2014-59084-P. Support for this work was provided by NASA through Hubble Fellowship grant \#HST-HF251357.001-A. MRS thanks for support from FONDECYT (1141269) and Millennium Science Initiative, Chilean ministry of Economy: Nucleus P10-022-F. This work has made use of data obtained at the Thai National Observatory on Doi Inthanon, operated by NARIT. The results presented in this paper are based on observations collected at the European Southern Observatory under programme IDs 086.D0161, 086.D-0265 and 192.D-0270. We thank Alex Gianninas for computing white dwarf limb darkening parameters for the $K G 5$ filter.

\section{REFERENCES}

Althaus L. G., Córsico A. H., Bischoff-Kim A., Romero A. D., Renedo I., García-Berro E., Miller Bertolami M. M., 2010,
ApJ, 717, 897

Baraffe I., Chabrier G., 1996, ApJ, 461, L51

Barstow M. A., Bond H. E., Holberg J. B., Burleigh M. R., Hubeny I., Koester D., 2005, MNRAS, 362, 1134

Benvenuto O. G., Althaus L. G., 1999, MNRAS, 303, 30

Bergeron P., Wesemael F., Fontaine G., 1991, ApJ, 367, 253

Bergeron P., Saffer R. A., Liebert J., 1992, ApJ, 394, 228

Bergeron P., Leggett S. K., Ruiz M. T., 2001, ApJS, 133, 413

Bond H. E., et al., 2015, ApJ, 813, 106

Bours M. C. P., et al., 2014, MNRAS, 438, 3399

Castanheira B. G., Kepler S. O., 2009, MNRAS, 396, 1709

Catalán S., Isern J., García-Berro E., Ribas I., 2008, MNRAS, 387, 1693

Chandrasekhar S., 1931, ApJ, 74, 81

Claret A., 2000, VizieR Online Data Catalog, 336, 31081

Claret A., Bloemen S., 2011, A\&A, 529, A75

Copperwheat C. M., Marsh T. R., Dhillon V. S., Littlefair S. P., Hickman R., Gänsicke B. T., Southworth J., 2010, MNRAS, 402, 1824

Dhillon V. S., et al., 2007, MNRAS, 378, 825

Dhillon V. S., et al., 2014, MNRAS, 444, 4009

Drake A. J., et al., 2009, ApJ, 696, 870

Drake A. J., et al., 2010, preprint, (arXiv:1009.3048)

Drake A. J., et al., 2014, ApJS, 213, 9

Finley D. S., Koester D., Basri G., 1997, ApJ, 488, 375

Fontaine G., Brassard P., Bergeron P., 2001, PASP, 113, 409

García-Berro E., Oswalt T. D., 2016, New Astron. Rev., 72, 1

Gianninas A., Strickland B. D., Kilic M., Bergeron P., 2013, ApJ, 766,3

Han Z., Tout C. A., Eggleton P. P., 2000, MNRAS, 319, 215

Hardy L. K., et al., 2017, MNRAS, 465, 4968

Hermes J. J., et al., 2015, MNRAS, 451, 1701

Holberg J. B., Oswalt T. D., Barstow M. A., 2012, AJ, 143, 68

Irawati P., et al., 2016, MNRAS, 456, 2446

Kepler S. O., Kleinman S. J., Nitta A., Koester D., Castanheira B. G., Giovannini O., Costa A. F. M., Althaus L., 2007, MNRAS, 375, 1315

Kleinman S. J., et al., 2004, ApJ, 607, 426

Koester D., 2010, Mem. Soc. Astron. Italiana, 81, 921

Krzeminski W., 1984, IAU Circ., 4014

Littlefair S. P., Dhillon V. S., Marsh T. R., Gänsicke B. T., Southworth J., Baraffe I., Watson C. A., Copperwheat C., 2008, MNRAS, 388, 1582

Marsh T. R., 1995, MNRAS, 275, L1

Marsh T. R., Robinson E. L., Wood J. H., 1994, MNRAS, 266, 137

Maxted P. F. L., O'Donoghue D., Morales-Rueda L., Napiwotzki R., Smalley B., 2007, MNRAS, 376, 919

Morris S. L., Naftilan S. A., 1993, ApJ, 419, 344

O'Brien M. S., Bond H. E., Sion E. M., 2001, ApJ, 563, 971

Panei J. A., Althaus L. G., Chen X., Han Z., 2007, MNRAS, 382,779

Parsons S. G., Marsh T. R., Copperwheat C. M., Dhillon V. S., Littlefair S. P., Gänsicke B. T., Hickman R., 2010, MNRAS, 402, 2591

Parsons S. G., et al., 2012a, MNRAS, 419, 304

Parsons S. G., et al., 2012b, MNRAS, 420, 3281

Parsons S. G., et al., 2012c, MNRAS, 426, 1950

Parsons S. G., et al., 2013, MNRAS, 429, 256

Parsons S. G., et al., 2015, MNRAS, 449, 2194

Parsons S. G., et al., 2016, MNRAS, 458, 2793

Perlmutter S., et al., 1999, ApJ, 517, 565

Prada Moroni P. G., Straniero O., 2009, A\&A, 507, 1575

Press W. H., Teukolsky A. A., Vetterling W. T., Flannery B. P., 2007, Numerical recipes. The art of scientific computing, 3rd edn.. Cambridge: University Press

Pyrzas S., et al., 2009, MNRAS, 394, 978

Pyrzas S., et al., 2012, MNRAS, 419, 817 
Rebassa-Mansergas A., Gänsicke B. T., Rodríguez-Gil P., Schreiber M. R., Koester D., 2007, MNRAS, 382, 1377

Rebassa-Mansergas A., Nebot Gómez-Morán A., Schreiber M. R., Girven J., Gänsicke B. T., 2011, MNRAS, 413, 1121

Rebassa-Mansergas A., Nebot Gómez-Morán A., Schreiber M. R., Gänsicke B. T., Schwope A., Gallardo J., Koester D., 2012, MNRAS, 419, 806

Rebassa-Mansergas A., Ren J. J., Parsons S. G., Gänsicke B. T., Schreiber M. R., García-Berro E., Liu X.-W., Koester D., 2016, MNRAS, 458, 3808

Ribeiro T., Baptista R., Kafka S., Dufour P., Gianninas A., Fontaine G., 2013, A\&A, 556, A34

Riess A. G., et al., 1998, AJ, 116, 1009

Romero A. D., Córsico A. H., Althaus L. G., Kepler S. O., Castanheira B. G., Miller Bertolami M. M., 2012, MNRAS, 420, 1462

Savoury C. D. J., et al., 2011, MNRAS, 415, 2025

Shipman H. L., Provencal J. L., Høg E., Thejll P., 1997, ApJ, 488, L43

Subasavage J. P., Jao W.-C., Henry T. J., Bergeron P., Dufour P., Ianna P. A., Costa E., Méndez R. A., 2009, AJ, 137, 4547

Tappert C., Gänsicke B. T., Rebassa-Mansergas A., Schmidtobreick L., Schreiber M. R., 2011, A\&A, 531, A113

Torres G., Andersen J., Giménez A., 2010, A\&ARv, 18, 67

Tremblay P.-E., Bergeron P., Kalirai J. S., Gianninas A., 2010, ApJ, 712, 1345

Tremblay P.-E., Ludwig H.-G., Steffen M., Freytag B., 2013, A\&A, 559, A104

Tremblay P.-E., et al., 2017, MNRAS, 465, 2849

Verbunt F., Rappaport S., 1988, ApJ, 332, 193

Vernet J., et al., 2011, A\&A, 536, A105

Willems B., Kolb U., 2004, A\&A, 419, 1057

Wood J. H., Irwin M. J., Pringle J. E., 1985, MNRAS, 214, 475

Zahn J.-P., 1977, A\&A, 57, 383

Zucker S., Mazeh T., Alexander T., 2007, ApJ, 670, 1326

\section{APPENDIX A: JOURNAL OF PHOTOMETRIC OBSERVATION}

\section{APPENDIX B: JOURNAL OF SPECTROSCOPIC OBSERVATIONS}

This paper has been typeset from a $\mathrm{T}_{\mathrm{E}} \mathrm{X} / \mathrm{LAT}_{\mathrm{E} X} \mathrm{X}$ file prepared by the author. 
Table A1. Journal of photometric observations.

\begin{tabular}{|c|c|c|c|c|c|c|c|c|}
\hline $\begin{array}{l}\text { Date at } \\
\text { start of run }\end{array}$ & Instrument & Telescope & Filter(s) & $\begin{array}{l}\text { Start } \\
\text { (UT) }\end{array}$ & $\begin{array}{l}\text { Orbital } \\
\text { phase }\end{array}$ & $\begin{array}{l}\text { Exposure } \\
\text { time }(\mathrm{s})\end{array}$ & $\begin{array}{l}\text { Number of } \\
\text { exposures }\end{array}$ & $\begin{array}{c}\text { Conditions } \\
\text { (Transparency, seeing) }\end{array}$ \\
\hline \multicolumn{9}{|c|}{ CSS 080502: } \\
\hline 2010-04-21 & ULTRACAM & NTT & $u^{\prime} g^{\prime} i^{\prime}$ & 23:48:00 & $0.90-1.60$ & 3.0 & 3339 & Good, 2 arcsec \\
\hline 2010-04-22 & ULTRACAM & NTT & $u^{\prime} g^{\prime} i^{\prime}$ & $23: 25: 47$ & $0.48-0.92$ & 2.0 & 2734 & Good, $\sim 1.5$ arcsec \\
\hline 2010-04-25 & ULTRACAM & NTT & $u^{\prime} g^{\prime} i^{\prime}$ & 01:04:33 & $0.33-0.65$ & 2.0 & 2066 & Average, $\sim 1.5$ arcsec \\
\hline 2010-11-23 & ULTRACAM & NTT & $u^{\prime} g^{\prime} i^{\prime}$ & 07:35:09 & $0.79-1.16$ & 3.7 & 1269 & Good, 1.5 arcsec \\
\hline 2010-11-26 & ULTRACAM & NTT & $u^{\prime} g^{\prime} i^{\prime}$ & $07: 10: 17$ & $0.75-1.13$ & 3.8 & 1269 & Excellent, $<1 \operatorname{arcsec}$ \\
\hline 2010-11-27 & ULTRACAM & NTT & $u^{\prime} g^{\prime} i^{\prime}$ & $06: 15: 06$ & $0.19-0.54$ & 3.7 & 1194 & Excellent, $<1$ arcsec \\
\hline 2010-12-15 & ULTRACAM & NTT & $u^{\prime} g^{\prime} i^{\prime}$ & $06: 52: 44$ & $0.82-1.29$ & 3.7 & 1583 & Good, 1 arcsec \\
\hline 2010-12-17 & ULTRACAM & NTT & $u^{\prime} g^{\prime} i^{\prime}$ & $05: 32: 20$ & $0.83-1.70$ & 3.7 & 2964 & Good, 1 arcsec \\
\hline 2016-11-10 & ULTRACAM & NTT & $u^{\prime} g^{\prime} r^{\prime}$ & 06:39:41 & $0.82-1.10$ & 3.0 & 1198 & Excellent, $<1$ arcsec \\
\hline \multicolumn{9}{|c|}{ CSS 09704: } \\
\hline 2010-11-29 & ULTRACAM & NTT & $u^{\prime} g^{\prime} i^{\prime}$ & $00: 24: 08$ & $0.85-1.22$ & 6.9 & 740 & Average, $\sim 1.5$ arcsec \\
\hline 2011-05-27 & ULTRACAM & NTT & $u^{\prime} g^{\prime} r^{\prime}$ & 09:02:36 & $0.88-1.28$ & 5.8 & 922 & Good, 1 arcsec \\
\hline 2011-11-01 & ULTRACAM & WHT & $u^{\prime} g^{\prime} r^{\prime}$ & 22:04:39 & $0.91-1.07$ & 4.1 & 531 & Excellent, $<1$ arcsec \\
\hline 2012-09-05 & ULTRACAM & WHT & $u^{\prime} g^{\prime} r^{\prime}$ & $20: 37: 30$ & $0.91-1.05$ & 3.2 & 517 & Good, 1 arcsec \\
\hline 2012-09-06 & ULTRACAM & WHT & $u^{\prime} g^{\prime} r^{\prime}$ & $22: 45: 51$ & $0.87-1.08$ & 3.3 & 834 & Average, $\sim 1.5$ arcsec \\
\hline 2012-10-09 & ULTRACAM & WHT & $u^{\prime} g^{\prime} r^{\prime}$ & 19:43:44 & $0.90-1.11$ & 3.1 & 887 & Good, $\sim 1.5$ arcsec \\
\hline 2016-11-11 & ULTRACAM & NTT & $u^{\prime} g^{\prime} r^{\prime}$ & 01:16:02 & $0.95-1.05$ & 4.0 & 318 & Good, 1 $\operatorname{arcsec}$ \\
\hline \multicolumn{9}{|c|}{ CSS 21357: } \\
\hline 2014-01-27 & ULTRASPEC & TNT & $K G 5$ & $19: 32: 23$ & $0.92-1.10$ & 1.5 & 2484 & Excellent, $\sim 1.5 \operatorname{arcsec}$ \\
\hline $2015-02-28$ & ULTRASPEC & TNT & $K G 5$ & $19: 26: 52$ & $0.94-1.09$ & 2.0 & 1584 & Good, $\sim 2 \operatorname{arcsec}$ \\
\hline 2015-03-01 & ULTRASPEC & TNT & KG5 & $19: 18: 13$ & $0.94-1.09$ & 2.0 & 1670 & Good, $\sim 1.5 \operatorname{arcsec}$ \\
\hline \multicolumn{9}{|c|}{ CSS 40190: } \\
\hline 2010-12-13 & ULTRACAM & NTT & $u^{\prime} g^{\prime} r^{\prime}$ & 07:41:50 & $0.89-1.14$ & 4.8 & 581 & Good, $\sim 2$ arcsec \\
\hline $\begin{array}{l}\text { 2016-11-11 } \\
\text { RR Cae: }\end{array}$ & \multicolumn{7}{|c|}{ RR Cae: } & Excellent, $<1$ arcsec \\
\hline $2005-11-25$ & ULTRACAM & VLT & $u^{\prime} g^{\prime} i^{\prime}$ & $00: 23: 57$ & $0.43-0.56$ & 0.5 & 6974 & Excellent, $<1 \operatorname{arcsec}$ \\
\hline 2005-11-27 & ULTRACAM & VLT & $u^{\prime} g^{\prime} i^{\prime}$ & $00: 02: 27$ & $0.96-1.06$ & 0.5 & 5061 & Excellent, $<1$ arcsec \\
\hline $2005-11-27$ & ULTRACAM & VLT & $u^{\prime} g^{\prime} i^{\prime}$ & 07:04:42 & $0.93-1.10$ & 0.5 & 8617 & Excellent, $<1$ arcsec \\
\hline 2010-11-15 & ULTRACAM & NTT & $u^{\prime} g^{\prime} i^{\prime}$ & $03: 31: 35$ & $0.37-0.58$ & 3.0 & 1834 & Good, $\sim 1 \operatorname{arcsec}$ \\
\hline 2010-11-21 & ULTRACAM & NTT & $u^{\prime} g^{\prime} i^{\prime}$ & $05: 34: 49$ & $0.41-0.85$ & 3.0 & 3901 & Good, 1.5 arcsec \\
\hline 2010-11-22 & ULTRACAM & NTT & $u^{\prime} g^{\prime} r^{\prime}$ & $06: 07: 21$ & $0.78-1.09$ & 3.0 & 2794 & Good, $\sim 1.5 \operatorname{arcsec}$ \\
\hline 2010-11-26 & ULTRACAM & NTT & $u^{\prime} g^{\prime} i^{\prime}$ & $01: 43: 15$ & $0.34-0.56$ & 2.5 & 2149 & Excellent, $<1$ arcsec \\
\hline $2010-11-27$ & ULTRACAM & NTT & $u^{\prime} g^{\prime} i^{\prime}$ & $03: 54: 33$ & $0.94-1.05$ & 2.5 & 1216 & Excellent, $<1 \operatorname{arcsec}$ \\
\hline $2010-11-27$ & ULTRACAM & NTT & $u^{\prime} g^{\prime} i^{\prime}$ & $07: 37: 29$ & $0.45-0.55$ & 2.5 & 1128 & Excellent, $<1$ arcsec \\
\hline 2010-12-02 & ULTRACAM & NTT & $u^{\prime} g^{\prime} i^{\prime}$ & 04:09:15 & $0.43-0.57$ & 2.5 & 1433 & Good, $\sim 1.5 \operatorname{arcsec}$ \\
\hline 2010-12-10 & ULTRACAM & NTT & $u^{\prime} g^{\prime} i^{\prime}$ & $00: 43: 35$ & $0.30-0.62$ & 3.0 & 2762 & Good, $\sim 1.5 \operatorname{arcsec}$ \\
\hline $2010-12-15$ & ULTRACAM & NTT & $u^{\prime} g^{\prime} i^{\prime}$ & $05: 26: 25$ & $0.41-0.59$ & 2.8 & 1590 & Good, 1 $\operatorname{arcsec}$ \\
\hline 2016-11-08 & ULTRACAM & NTT & $u^{\prime} g^{\prime} r^{\prime}$ & $04: 37: 46$ & $0.98-1.04$ & 2.0 & 743 & Excellent, $<1$ arcsec \\
\hline \multicolumn{9}{|c|}{ SDSS J0024+1745: } \\
\hline 2015-09-18 & ULTRACAM & WHT & $u^{\prime} g^{\prime} r^{\prime}$ & $02: 57: 44$ & $0.91-1.10$ & 0.6 & 5966 & Average, $\sim 2$ arcsec \\
\hline 2016-11-08 & ULTRACAM & NTT & $u^{\prime} g^{\prime} r^{\prime}$ & $00: 13: 14$ & $0.92-1.07$ & 4.0 & 688 & Excellent, $<1$ arcsec \\
\hline 2016-11-09 & ULTRACAM & NTT & $u^{\prime} g^{\prime} r^{\prime}$ & $00: 31: 37$ & 0.98-1.09 & 4.0 & 484 & Good, 1 $\operatorname{arcsec}$ \\
\hline \multicolumn{9}{|c|}{ SDSS J0106-0014: } \\
\hline 2010-11-11 & ULTRACAM & NTT & $u^{\prime} g^{\prime} i^{\prime}$ & $00: 22: 51$ & $0.28-1.64$ & 4.0 & 2494 & Good, $\sim 1$ arcsec \\
\hline $2010-11-23$ & ULTRACAM & NTT & $u^{\prime} g^{\prime} i^{\prime}$ & $00: 30: 57$ & $0.48-0.60$ & 5.0 & 1949 & Good, 1.5 $\operatorname{arcsec}$ \\
\hline 2010-11-26 & ULTRACAM & NTT & $u^{\prime} g^{\prime} i^{\prime}$ & $00: 40: 45$ & $0.85-1.25$ & 2.5 & 1159 & Excellent, $<1$ arcsec \\
\hline 2010-11-26 & ULTRACAM & NTT & $u^{\prime} g^{\prime} i^{\prime}$ & $04: 22: 13$ & $0.66-1.20$ & 4.0 & 996 & Excellent, $<1 \operatorname{arcsec}$ \\
\hline $2010-11-27$ & ULTRACAM & NTT & $u^{\prime} g^{\prime} i^{\prime}$ & $00: 29: 50$ & $0.52-1.13$ & 2.5 & 1868 & Excellent, $<1$ arcsec \\
\hline $2010-12-17$ & ULTRACAM & NTT & $u^{\prime} g^{\prime} i^{\prime}$ & $00: 42: 45$ & $0.86-1.14$ & 3.0 & 671 & Good, $\sim 1$ arcsec \\
\hline 2011-11-01 & ULTRACAM & WHT & $u^{\prime} g^{\prime} r^{\prime}$ & 20:30:02 & $0.86-1.24$ & 4.0 & 703 & Excellent, $<1$ arcsec \\
\hline 2011-11-02 & ULTRACAM & WHT & $u^{\prime} g^{\prime} r^{\prime}$ & $20: 59: 54$ & $0.87-1.22$ & 3.0 & 843 & Average, $\sim 1.5$ arcsec \\
\hline 2012-09-06 & ULTRACAM & WHT & $u^{\prime} g^{\prime} r^{\prime}$ & $23: 40: 07$ & $0.81-1.18$ & 4.0 & 753 & Good, 1.5 arcsec \\
\hline 2012-09-07 & ULTRACAM & WHT & $u^{\prime} g^{\prime} r^{\prime}$ & 01:40:32 & $0.80-1.04$ & 2.0 & 926 & Excellent, $<1 \operatorname{arcsec}$ \\
\hline 2012-10-10 & ULTRACAM & WHT & $u^{\prime} g^{\prime} r^{\prime}$ & $21: 57: 26$ & $0.91-1.08$ & 3.1 & 401 & Excellent, $<1$ arcsec \\
\hline 2016-11-08 & ULTRACAM & NTT & $u^{\prime} g^{\prime} r^{\prime}$ & 01:04:45 & $0.92-1.07$ & 4.0 & 278 & Excellent, $<1$ arcsec \\
\hline \multicolumn{9}{|c|}{ SDSS J0110+1326: } \\
\hline 2007-10-21 & ULTRACAM & WHT & $u^{\prime} g^{\prime} i^{\prime}$ & $02: 51: 57$ & $0.86-1.07$ & 1.2 & 4805 & Good, 1.5 arcsec \\
\hline 2010-11-23 & ULTRACAM & NTT & $u^{\prime} g^{\prime} i^{\prime}$ & $02: 57: 19$ & $0.45-0.56$ & 3.0 & 1140 & Good, 1.5 arcsec \\
\hline 2012-09-09 & ULTRACAM & WHT & $u^{\prime} g^{\prime} i^{\prime}$ & $00: 35: 56$ & $0.97-1.04$ & 2.0 & 858 & Good, 1 $\operatorname{arcsec}$ \\
\hline 2012-10-09 & ULTRACAM & WHT & $u^{\prime} g^{\prime} r^{\prime}$ & 22:39:02 & $0.91-1.04$ & 2.0 & 1829 & Good, $\sim 2$ arcsec \\
\hline 2015-09-19 & ULTRACAM & WHT & $u^{\prime} g^{\prime} r^{\prime}$ & 04:33:21 & $0.91-1.02$ & 1.8 & 1764 & Average, $\sim 1.5$ arcsec \\
\hline 2016-11-08 & ULTRACAM & NTT & $u^{\prime} g^{\prime} r^{\prime}$ & $01: 30: 59$ & $0.97-1.03$ & 2.0 & 936 & Excellent, $<1$ arcsec \\
\hline
\end{tabular}




\begin{tabular}{|c|c|c|c|c|c|c|c|c|}
\hline $\begin{array}{l}\text { Date at } \\
\text { start of run }\end{array}$ & Instrument & Telescope & Filter $(\mathrm{s})$ & $\begin{array}{l}\text { Start } \\
(\mathrm{UT})\end{array}$ & $\begin{array}{l}\text { Orbital } \\
\text { phase }\end{array}$ & $\begin{array}{l}\text { Exposure } \\
\text { time }(\mathrm{s})\end{array}$ & $\begin{array}{l}\text { Number of } \\
\text { exposures }\end{array}$ & $\begin{array}{c}\text { Conditions } \\
\text { (Transparency, seeing) }\end{array}$ \\
\hline \multicolumn{9}{|c|}{ SDSS J0314+0206: } \\
\hline 2012-10-14 & ULTRACAM & WHT & $u^{\prime} g^{\prime} r^{\prime}$ & $02: 42: 14$ & $0.94-1.05$ & 1.6 & 1712 & Good, $\sim 1.5$ arcsec \\
\hline 2016-11-09 & ULTRACAM & NTT & $u^{\prime} g^{\prime} r^{\prime}$ & 05:00:17 & $0.93-1.07$ & 3.5 & 1040 & Excellent, $<1$ arcsec \\
\hline \multicolumn{9}{|c|}{ SDSS J1021+1744: } \\
\hline 2015-01-17 & ULTRACAM & WHT & $u^{\prime} g^{\prime} r^{\prime}$ & $01: 12: 30$ & $0.78-1.29$ & 4.0 & 1563 & Good, $\sim 1$ arcsec \\
\hline 2015-05-19 & ULTRACAM & WHT & $u^{\prime} g^{\prime} z^{\prime}$ & $21: 13: 23$ & $0.89-1.09$ & 9.9 & 245 & Good, $\sim 2$ arcsec \\
\hline $2015-05-20$ & ULTRACAM & WHT & $u^{\prime} g^{\prime} i^{\prime}$ & $21: 11: 18$ & $0.01-0.48$ & 5.0 & 1160 & Good, $\sim 1.5$ arcsec \\
\hline \multicolumn{9}{|c|}{ SDSS J1028+0931: } \\
\hline 2014-11-27 & ULTRASPEC & TNT & $g^{\prime}$ & $21: 14: 20$ & $0.90-1.06$ & 1.0 & 3163 & Good, $\sim 1.5$ arcsec \\
\hline 2014-12-01 & ULTRASPEC & TNT & $g^{\prime}$ & $20: 48: 21$ & $0.85-1.04$ & 1.0 & 3943 & Good, $\sim 2$ arcsec \\
\hline 2015-01-06 & ULTRASPEC & TNT & $g^{\prime}$ & $20: 08: 27$ & $0.92-1.07$ & 1.0 & 3056 & Good, $\sim 2$ arcsec \\
\hline $2015-02-25$ & ULTRASPEC & TNT & $g^{\prime}$ & $15: 33: 21$ & $0.86-1.07$ & 1.0 & 4377 & Good, $\sim 2 \operatorname{arcsec}$ \\
\hline 2015-03-03 & ULTRASPEC & TNT & $g^{\prime}$ & $18: 21: 00$ & $0.88-1.09$ & 1.0 & 4199 & Good, $\sim 1.5$ arcsec \\
\hline 2016-03-17 & ULTRASPEC & TNT & $g^{\prime}$ & 19:02:34 & $0.85-1.05$ & 1.0 & 4091 & Average, $\sim 1.5$ arcsec \\
\hline \multicolumn{9}{|c|}{ SDSS J1123-1155: } \\
\hline 2014-01-28 & ULTRASPEC & TNT & $K G 5$ & $17: 19: 39$ & $0.92-1.04$ & 1.5 & 5404 & Good, $\sim 1.5$ arcsec \\
\hline \multicolumn{9}{|c|}{ SDSS J1307+2156: } \\
\hline $2015-02-28$ & ULTRASPEC & TNT & $K G 5$ & $21: 18: 08$ & $0.91-1.05$ & 4.8 & 542 & Good, $\sim 2$ arcsec \\
\hline \multicolumn{9}{|c|}{ SDSS J1329+1230: } \\
\hline 2010-04-21 & ULTRACAM & NTT & $u^{\prime} g^{\prime} i^{\prime}$ & $05: 18: 12$ & $0.76-1.50$ & 3.0 & 1738 & Excellent, $<1$ arcsec \\
\hline 2010-04-22 & ULTRACAM & NTT & $u^{\prime} g^{\prime} i^{\prime}$ & $03: 44: 44$ & $0.31-0.53$ & 3.8 & 410 & Good, $\sim 2$ arcsec \\
\hline 2010-04-22 & ULTRACAM & NTT & $u^{\prime} g^{\prime} i^{\prime}$ & $05: 20: 42$ & $0.13-0.70$ & 3.8 & 1041 & Good, $\sim 2 \operatorname{arcsec}$ \\
\hline 2010-04-23 & ULTRACAM & NTT & $u^{\prime} g^{\prime} i^{\prime}$ & $01: 06: 14$ & $0.30-1.12$ & 3.8 & 1496 & Good, $\sim 1.5$ arcsec \\
\hline 2010-04-23 & ULTRACAM & NTT & $u^{\prime} g^{\prime} i^{\prime}$ & 04:05:44 & $0.85-1.13$ & 3.8 & 531 & Good, $\sim 1.5$ arcsec \\
\hline 2010-04-23 & ULTRACAM & NTT & $u^{\prime} g^{\prime} i^{\prime}$ & 06:01:07 & $0.83-1.30$ & 3.8 & 861 & Good, 1.5 arcsec \\
\hline 2010-04-25 & ULTRACAM & NTT & $u^{\prime} g^{\prime} i^{\prime}$ & $04: 49: 24$ & $0.91-1.65$ & 3.9 & 1315 & Average, $\sim 1.5$ arcsec \\
\hline $2010-04-28$ & ULTRACAM & NTT & $u^{\prime} g^{\prime} r^{\prime}$ & $02: 48: 35$ & $0.93-1.15$ & 3.9 & 404 & Good, $\sim 1.5$ arcsec \\
\hline \multicolumn{9}{|c|}{ SDSS J2235+1428: } \\
\hline 2010-11-12 & ULTRACAM & NTT & $u^{\prime} g^{\prime} i^{\prime}$ & $00: 14: 36$ & $0.31-1.08$ & 5.0 & 1933 & Good, $\sim 1$ arcsec \\
\hline $2010-11-15$ & ULTRACAM & NTT & $u^{\prime} g^{\prime} i^{\prime}$ & $01: 06: 38$ & $0.32-0.61$ & 5.0 & 717 & Good, $\sim 2$ arcsec \\
\hline 2011-11-01 & ULTRACAM & WHT & $u^{\prime} g^{\prime} r^{\prime}$ & $21: 20: 33$ & $0.96-1.13$ & 5.0 & 351 & Excellent, $<1$ arcsec \\
\hline 2012-09-07 & ULTRACAM & WHT & $u^{\prime} g^{\prime} r^{\prime}$ & 00:33:05 & $0.87-1.06$ & 4.0 & 598 & Good, $\sim 1.5$ arcsec \\
\hline 2012-09-10 & ULTRACAM & WHT & $u^{\prime} g^{\prime} r^{\prime}$ & $21: 57: 26$ & $0.81-1.09$ & 4.0 & 851 & Good, $\sim 2$ arcsec \\
\hline 2015-09-19 & ULTRACAM & WHT & $u^{\prime} g^{\prime} r^{\prime}$ & $20: 44: 26$ & $0.89-1.06$ & 4.0 & 551 & Average, $\sim 2$ arcsec \\
\hline 2016-11-09 & ULTRACAM & NTT & $u^{\prime} g^{\prime} r^{\prime}$ & $01: 14: 41$ & $0.91-1.08$ & 4.1 & 512 & Excellent, $<1$ arcsec \\
\hline \multicolumn{9}{|c|}{ WD 1333+005: } \\
\hline 2014-02-02 & ULTRASPEC & TNT & $K G 5$ & $18: 54: 41$ & $0.84-1.13$ & 1.5 & 1998 & Good, $\sim 1.5$ arcsec \\
\hline $2015-02-23$ & ULTRASPEC & TNT & $K G 5$ & $18: 28: 12$ & $0.71-1.10$ & 3.0 & 1384 & Good, $\sim 1.5$ arcsec \\
\hline 2015-03-30 & ULTRASPEC & TNT & $K G 5$ & $15: 59: 01$ & $0.86-1.09$ & 3.5 & 688 & Good, $\sim 2$ arcsec \\
\hline 2016-03-19 & ULTRASPEC & TNT & $K G 5$ & $16: 13: 42$ & $0.76-1.10$ & 4.0 & 900 & Average, $\sim 2$ arcsec \\
\hline
\end{tabular}


Table B1. Journal of X-shooter spectroscopic observations. We have not included the NIR exposure times or number of exposures for objects with very low signal-to-noise ratios in this arm, since this data was discarded.

\begin{tabular}{|c|c|c|c|c|c|}
\hline $\begin{array}{l}\text { Date at } \\
\text { start of run }\end{array}$ & $\begin{array}{l}\text { Start } \\
\text { (UT) }\end{array}$ & $\begin{array}{l}\text { Orbital } \\
\text { phase }\end{array}$ & $\begin{array}{l}\text { Exposure time (s) } \\
\text { UVB/VIS/NIR }\end{array}$ & $\begin{array}{c}\text { Number of exposures } \\
\text { UVB/VIS/NIR }\end{array}$ & $\begin{array}{c}\text { Conditions } \\
\text { (Transparency, seeing) }\end{array}$ \\
\hline \multicolumn{6}{|c|}{ CSS 080502: } \\
\hline 2014-03-04 & $01: 18: 17$ & $0.07-0.39$ & $368 / 410 / 300$ & $10 / 9 / 13$ & Good, 1 $\operatorname{arcsec}$ \\
\hline 2014-03-05 & $00: 30: 13$ & $0.54-0.98$ & $368 / 418 / 300$ & $14 / 12 / 18$ & Good, $\sim 1.5 \operatorname{arcsec}$ \\
\hline \multicolumn{6}{|c|}{ CSS 09704: } \\
\hline 2013-10-25 & $00: 23: 19$ & $0.16-0.40$ & $600 / 638 /-$ & $6 / 6 /-$ & Good, $\sim 1 \operatorname{arcsec}$ \\
\hline $2013-10-27$ & $00: 31: 05$ & $0.98-1.07$ & $600 / 638 /-$ & $3 / 3 /-$ & Excellent, $<1$ arcsec \\
\hline 2014-10-30 & 01:02:06 & $0.46-0.94$ & $600 / 600 /-$ & $10 / 10 /-$ & Excellent, $<1$ arcsec \\
\hline \multicolumn{6}{|c|}{ CSS 21357: } \\
\hline 2014-04-21 & $03: 12: 16$ & $0.30-1.02$ & $600 / 301 / 200$ & $23 / 42 / 71$ & Excellent, $<1$ arcsec \\
\hline 2014-04-22 & $02: 28: 15$ & $0.20-0.32$ & $600 / 301 / 200$ & $5 / 9 / 15$ & Excellent, $<1$ arcsec \\
\hline \multicolumn{6}{|c|}{ CSS 40190: } \\
\hline 2014-03-06 & $00: 18: 40$ & $0.92-1.70$ & $530 / 565 /-$ & 10/10/- & Good, 1.5 $\operatorname{arcsec}$ \\
\hline 2014-03-07 & $00: 18: 21$ & $0.60-1.45$ & $495 / 530 /-$ & $16 / 16 /-$ & Good, $\sim 1.5 \operatorname{arcsec}$ \\
\hline \multicolumn{6}{|c|}{ SDSS J0024+1745: } \\
\hline $2013-10-25$ & 04:16:41 & $0.87-0.99$ & $606 / 294 / 100$ & $4 / 7 / 23$ & Good, $\sim 1 \operatorname{arcsec}$ \\
\hline $2013-10-26$ & $00: 35: 24$ & $0.10-0.91$ & $606 / 294 / 100$ & $21 / 39 / 108$ & Good, 1.5 $\operatorname{arcsec}$ \\
\hline 2014-10-31 & 01:44:41 & $0.99-1.07$ & $600 / 300 / 100$ & $3 / 5 / 17$ & Excellent, $<1$ arcsec \\
\hline \multicolumn{6}{|c|}{ SDSS J0106-0014: } \\
\hline 2013-10-27 & 02:11:21 & $0.52-1.70$ & $480 / 515 /-$ & $16 / 16 /-$ & Good, $\sim 1.5 \operatorname{arcsec}$ \\
\hline \multicolumn{6}{|c|}{ SDSS J0110+1326: } \\
\hline 2010-10-01 & 03:08:20 & $0.17-0.24$ & $300 / 337 / 385$ & $6 / 6 / 6$ & Good, $\sim 1.5 \operatorname{arcsec}$ \\
\hline $2013-10-25$ & $03: 00: 25$ & $0.68-0.81$ & $300 / 338 / 386$ & $11 / 10 / 10$ & Good, 1 arcsec \\
\hline $2013-10-26$ & $04: 44: 29$ & $0.91-1.12$ & $300 / 338 / 386$ & $18 / 16 / 16$ & Good, $\sim 1.5$ arcsec \\
\hline $2013-10-27$ & 01:10:42 & $0.47-0.56$ & $300 / 338 / 386$ & $9 / 8 / 8$ & Good, 1.5 $\operatorname{arcsec}$ \\
\hline \multicolumn{6}{|c|}{ SDSS J0314+0206: } \\
\hline 2013-10-26 & $06: 42: 33$ & $0.36-0.64$ & $360 / 398 / 386$ & 19/17/19 & Good, 1.5 $\operatorname{arcsec}$ \\
\hline 2014-10-30 & 04:12:03 & $0.67-1.29$ & $410 / 400 / 440$ & $32 / 32 / 34$ & Excellent, $<1$ arcsec \\
\hline \multicolumn{6}{|c|}{ SDSS J1021+1744: } \\
\hline 2014-03-04 & 02:46:35 & $0.54-0.84$ & $645 / 615 / 300$ & $6 / 6 / 15$ & Good, $\sim 1.5 \operatorname{arcsec}$ \\
\hline 2014-03-05 & $02: 20: 18$ & $0.53-1.67$ & $645 / 615 / 300$ & $20 / 20 / 44$ & Good, 1.5 arcsec \\
\hline \multicolumn{6}{|c|}{ SDSS J1028+0931: } \\
\hline 2014-03-06 & $01: 51: 24$ & $0.71-1.26$ & $408 / 397 / 254$ & $19 / 19 / 33$ & Good, $\sim 1$ arcsec \\
\hline 2014-04-20 & $00: 28: 02$ & $0.92-1.09$ & $408 / 397 / 254$ & $8 / 8 / 14$ & Excellent, $<1$ arcsec \\
\hline 2014-04-22 & $00: 26: 59$ & $0.42-0.70$ & $408 / 397 / 254$ & $12 / 12 / 20$ & Excellent, $<1$ arcsec \\
\hline \multicolumn{6}{|c|}{ SDSS J1123-1155: } \\
\hline 2014-03-07 & $03: 20: 47$ & $0.57-0.65$ & $495 / 530 / 270$ & $10 / 10 / 20$ & Good, $1.5 \operatorname{arcsec}$ \\
\hline 2014-04-19 & 00:05:54 & $0.30-0.45$ & $495 / 530 / 270$ & $20 / 17 / 33$ & Good, 1 arcsec \\
\hline 2014-04-20 & $23: 39: 22$ & $0.87-1.05$ & $495 / 530 / 270$ & $21 / 19 / 40$ & Excellent, $<1$ arcsec \\
\hline 2014-04-21 & $23: 52: 26$ & $0.19-0.20$ & $495 / 530 / 270$ & $3 / 3 / 6$ & Excellent, $<1$ arcsec \\
\hline \multicolumn{6}{|c|}{ SDSS J1307+2156: } \\
\hline 2014-04-20 & 01:41:05 & $0.63-1.38$ & $400 / 300 / 100$ & $32 / 39 / 127$ & Excellent, $<1$ arcsec \\
\hline \multicolumn{6}{|c|}{ SDSS J1329+1230: } \\
\hline 2010-04-05 & 02:38:17 & $0.76-1.63$ & $300 / 338 /-$ & $16 / 16 /-$ & Good, $\sim 1.5$ arcsec \\
\hline \multicolumn{6}{|c|}{ SDSS J2235+1428: } \\
\hline 2010-09-30 & $23: 38: 14$ & $0.40-1.35$ & $300 / 337 /-$ & $27 / 27 /-$ & Good, $\sim 1.5 \operatorname{arcsec}$ \\
\hline \multicolumn{6}{|c|}{ WD 1333+005: } \\
\hline 2014-03-06 & 05:11:05 & $0.54-1.60$ & $366 / 408 / 300$ & $27 / 24 / 36$ & Good, $\sim 1$ arcsec \\
\hline
\end{tabular}

